BMC

Genomics

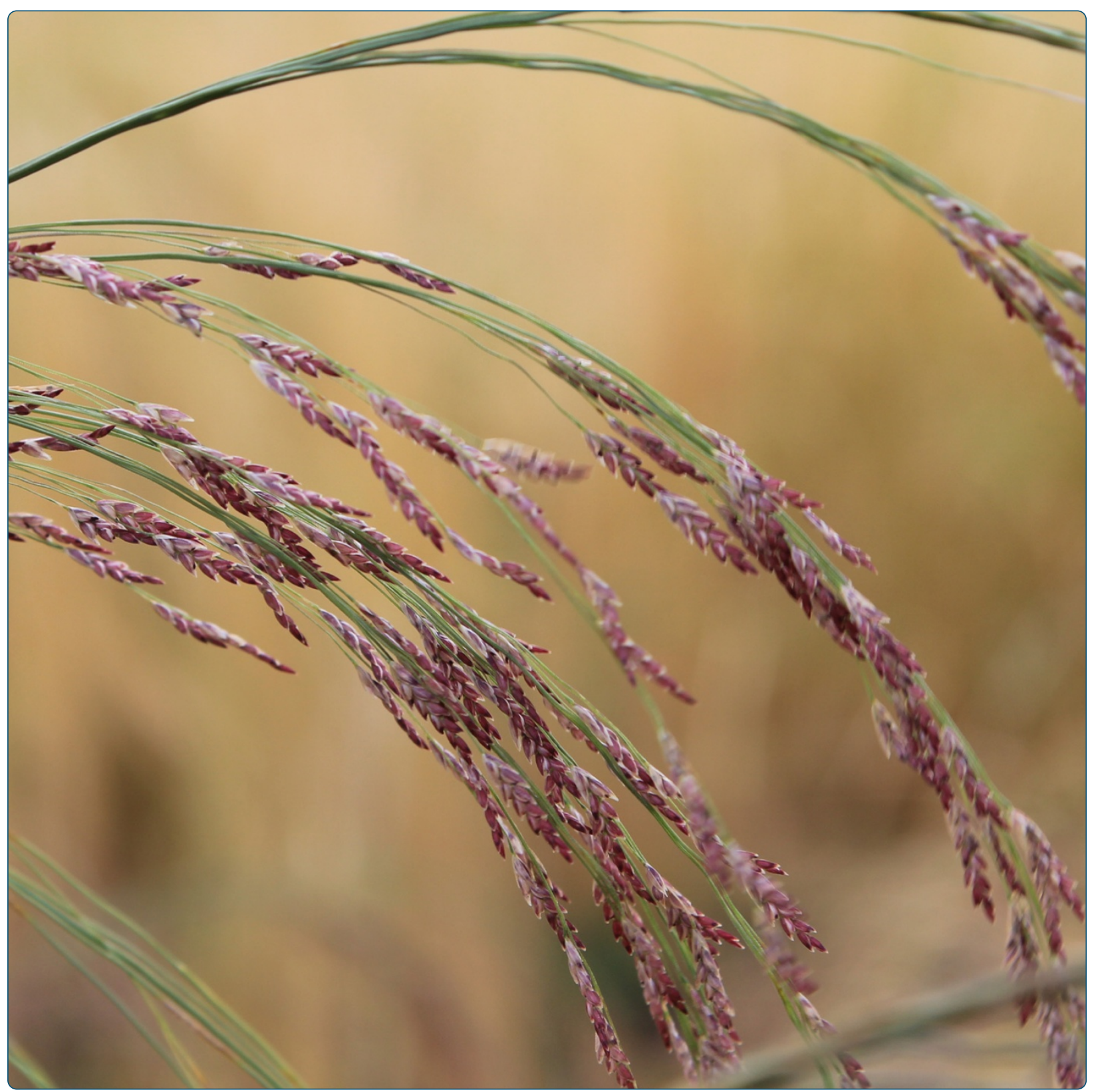

\title{
Genome and transcriptome sequencing identifies breeding targets in the orphan crop tef (Eragrostis tef)
}

Cannarozzi et al. 


\title{
Genome and transcriptome sequencing identifies breeding targets in the orphan crop tef (Eragrostis tef)
}

\author{
Gina Cannarozzi ${ }^{1,2}$, Sonia Plaza-Wüthrich ${ }^{1}$, Korinna Esfeld ${ }^{1}$, Stéphanie Larti ${ }^{1,8}$, Yi Song Wilson ${ }^{1}$, Dejene Girma ${ }^{1,3}$, \\ Edouard de Castro ${ }^{4}$, Solomon Chanyalew ${ }^{5}$, Regula Blösch', Laurent Farinelli ${ }^{6}$, Eric Lyons ${ }^{7}$, Michel Schneider ${ }^{4}$, \\ Laurent Falquet ${ }^{2,9}$, Cris Kuhlemeier ${ }^{1}$, Kebebew Assefa ${ }^{5}$ and Zerihun Tadele ${ }^{1 *}$
}

\begin{abstract}
Background: Tef (Eragrostis tef), an indigenous cereal critical to food security in the Horn of Africa, is rich in minerals and protein, resistant to many biotic and abiotic stresses and safe for diabetics as well as sufferers of immune reactions to wheat gluten. We present the genome of tef, the first species in the grass subfamily Chloridoideae and the first allotetraploid assembled de novo. We sequenced the tef genome for marker-assisted breeding, to shed light on the molecular mechanisms conferring tef's desirable nutritional and agronomic properties, and to make its genome publicly available as a community resource.
\end{abstract}

Results: The draft genome contains $672 \mathrm{Mbp}$ representing 87\% of the genome size estimated from flow cytometry. We also sequenced two transcriptomes, one from a normalized RNA library and another from unnormalized RNASeq data. The normalized RNA library revealed around 38000 transcripts that were then annotated by the SwissProt group. The CoGe comparative genomics platform was used to compare the tef genome to other genomes, notably sorghum. Scaffolds comprising approximately half of the genome size were ordered by syntenic alignment to sorghum producing tef pseudo-chromosomes, which were sorted into A and B genomes as well as compared to the genetic map of tef. The draft genome was used to identify novel SSR markers, investigate target genes for abiotic stress resistance studies, and understand the evolution of the prolamin family of proteins that are responsible for the immune response to gluten.

Conclusions: It is highly plausible that breeding targets previously identified in other cereal crops will also be valuable breeding targets in tef. The draft genome and transcriptome will be of great use for identifying these targets for genetic improvement of this orphan crop that is vital for feeding 50 million people in the Horn of Africa.

Keywords: Tef, Eragrostis tef, Genome, Transcriptome, Abiotic stress, Prolamin

\section{Background}

The increase in the global population, competition of food and biofuel for available land resources, and climate change are all threatening food security. One avenue to alleviate these pressures on our food supply is through better utilization of indigenous or 'orphan' crops. These crops have the advantages that they are already wellintegrated in the socio-economics of the region, they are

\footnotetext{
* Correspondence: zerihun.tadele@ips.unibe.ch

${ }^{1}$ University of Bern, Institute of Plant Sciences, Altenbergrain 21, Bern $\mathrm{CH}-3013$, Switzerland

Full list of author information is available at the end of the article
}

the preferred crops for both farmers and consumers, and they provide more stability under rapidly changing environmental conditions and demand. However, they have long been neglected both by commercial breeders and non-profit institutions. Tef [Eragrostis tef (Zucc.) Trotter] (family Poaceae, subfamily Chloridoideae) is an orphan cereal that is a staple food for over $70 \%$ of the 80 million people in Ethiopia where it grows annually on about 3 million hectares of land [1].

Tef is a prime candidate for genetic improvement both because of its nutritional and health benefits, and because of its tolerance to biotic and abiotic stresses, particularly 
drought and waterlogging. It can be cultivated in a wide range of ecological niches, including semi-arid areas prone to drought where maize, rice and wheat do not survive. While tef is the most important crop in Ethiopia, it is gaining in popularity as a life-style food in the developed world because it is gluten-free and high in protein, vitamins, and minerals such as calcium, iron and zinc [2-4].

Sensitivity to wheat, barley and rye gluten is related to the presence of specific epitopes in the prolamin gene family [5]. Tef has been proposed as a valuable addition to the diets of celiac patients [6] due to the absence of these epitopes as determined by antibody-based assays [7]. In addition, tef contains a high amount of 'slowly-digestible' starch conferring it with a low glycemic index (GI) and is considered a suitable food for Type 2 diabetics [3].

Relatively little sequence data are available for tef. A genetic map was constructed from 151 recombinant inbred lines (Eragrostis tef cv. Kaye Murri x Eragrostis pilosa) [8]. This data set includes 496 amplifiable simple sequence repeats (SSRs) of which 262 had at least one polymorphism and 192 were placed on 30 linkage groups. In addition, a collection of 3603 EST sequences also from the Kaye Murri cultivar are available [9]. The homologs of two genes involved in plant height (rht1 and sd1) have been cloned and sequenced for 31 cultivars [10].

Tef $(2 C=2 n=4 x=40)$ is an allotetraploid, the result of a genome duplication by hybridization between two diploid progenitors. Tef's closest relatives within the Eragrostis genus are thought to be E. pilosa and E. heteromera [11], although E. aethiopica, E. barrelieri, E. curvula and E. cilianensis may also be involved in the evolution of tef [12]. As both E. pilosa and E. heteromera are tetraploid species, the true diploid progenitors of tef remain unknown. The genome size of the Tsedey cultivar (DZ-Cr-37) sequenced here has been estimated as 772 Mbp by flow cytometry [13].

Whole genome duplication events create another copy of all nuclear genes and regulatory sequences at once, providing redundant gene copies for subsequent selection and adaptation. Ancient whole genome duplications are suggested to be associated with adaptive radiations [14] and contemporaneous with extinction events [15], supporting the idea that polyploidy is a driving force of plant evolution. Allotetraploidy, in particular, may contribute to the adaptability to novel and extreme environments [16] and may also increase the fitness of the polyploid in a given environment compared to its diploid progenitors [17]. As the tef genome is relatively small compared to other polyploid crop species, there is considerable interest in tef as both a model plant for polyploid genome evolution as well as for polyploid sequence assembly and analysis.

The Tef Improvement Project at the University of Bern in collaboration with the Ethiopian Institute of
Agricultural Research has taken the initiative to support the conventional breeding efforts in Ethiopia using modern molecular techniques. The overall objective of the project is to provide new cultivars improved in traits such as plant architecture, abiotic stress tolerance, and increased yield to subsistence farmers in Ethiopia in a timely manner. The genome and transcriptome sequences reported here reveal genes that have shaped a plant resilient to environmental stresses while also producing nutritious food.

\section{Results and discussion}

\section{Genome sequencing and assembly}

The general strategy of the tef genome and transcriptome sequencing, annotation and analysis is shown in Figure 1. The early-maturing improved variety of tef, Tsedey (DZ-Cr-37), was selected for genome sequencing as it can adapt to a wide variety of climates.

Homoeologous genomes in polyploids such as tef have high levels of sequence identity that present enormous challenges to assembly. Currently available genome assemblers are not designed to assemble polyploids and the resulting assemblies are often fragmented [18], chimeric [19] and/or contain false segmental duplications [20].

Often, strategies are applied to reduce the ploidy of the genome in order to simplify the assembly process. These include sequencing the diploid progenitors of a polyploid as was done for tobacco [21] and cotton [22], obtaining a haploid (the drone in ants) [23] or generating a doubled haploid. In plants, generating a double haploid can be done by producing a haploid genome from pollen or seeds and then doubling it to form a homozygous diploid, as done for the potato [24]. Alternatively, BAC libraries can be used to sequence and assemble the entire genome although this is time consuming and expensive.

Our attempts at obtaining a haploid tef for sequencing were unsuccessful. Hence, we sequenced allotetraploid tissue and expected to find a mixture of the A and B genomes. Multiple sequence alignments between the homeologous tef scaffolds and the few Sanger sequences for which we have both an A and a B copy do show some chimerism, for example, the KO2 gene (Additional file 1: Figure S1). However, the homeolog specificity is intact for large regions of the genes. A two-stage assembly strategy has been successful in separating homeologous sequences from the three genomes of wheat and could be applied to separate the homeologs for regions in which homeolog specificity is required [25].

We generated a total of $40 \mathrm{Gbp}$ from single- and pairedend reads resulting in 44-fold coverage with Illumina HiSeq2000 and seven-fold coverage with 454-FLX pyrosequencing. The libraries had insert sizes of $300 \mathrm{bp}, 3 \mathrm{~kb}$, $6.5 \mathrm{~kb}$ and $13 \mathrm{~kb}$ (Additional file 2: Table S1). The Illumina and the single-end 454-FLX sequences were assembled into contigs using SOAPdenovo [26]. The paired-end and 


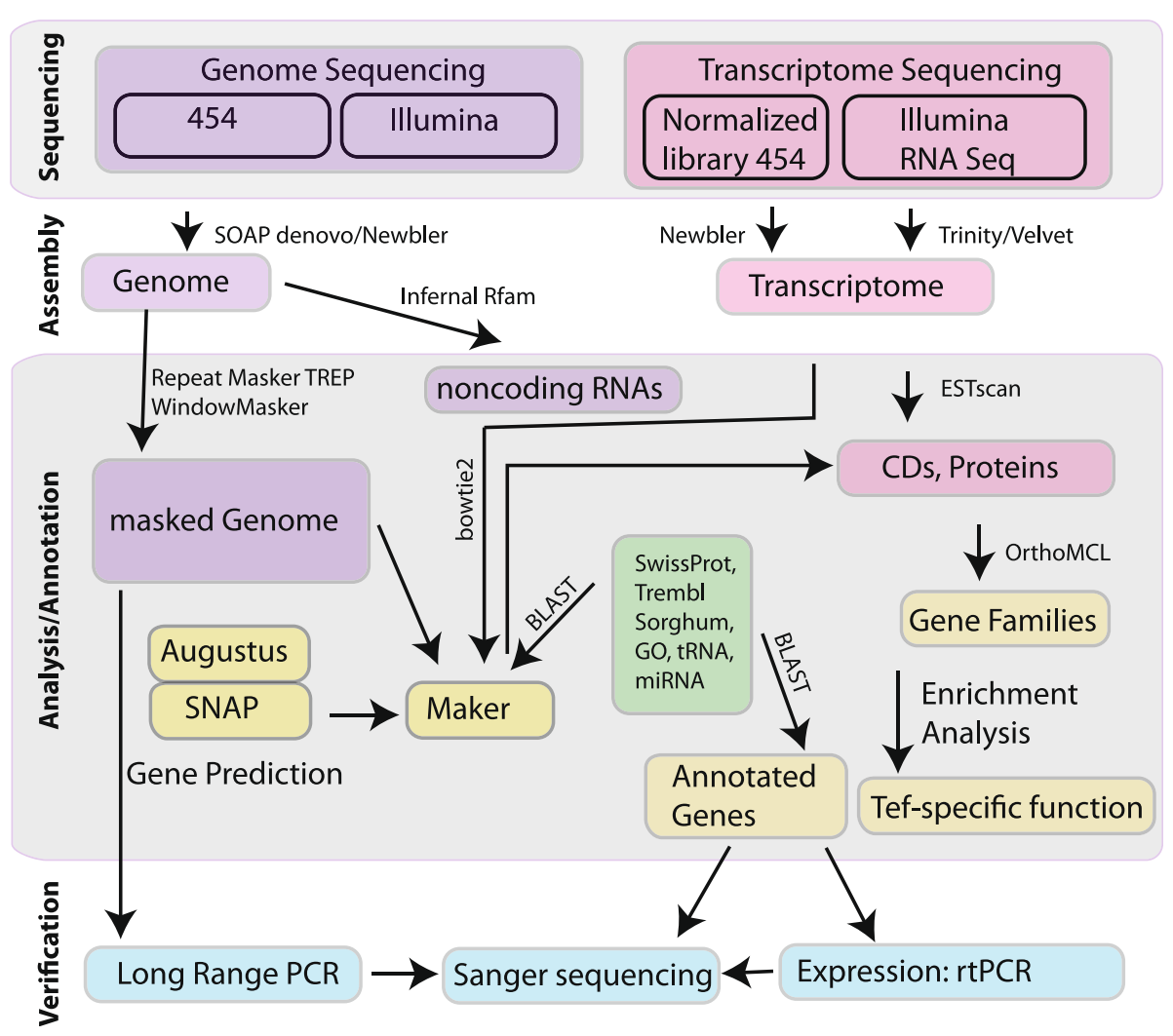

Figure 1 Overview of the tef sequencing project. Both the genome and transcriptome of tef were sequenced, annotated, analyzed and verified. The genome was assembled using SOAPdenovo and was then analyzed for transposable elements using WindowMasker, RepeatMasker and TREP. Non-coding RNAs were found with Infernal with the Rfam dataset and genes were predicted using the evidence combiner, Maker. One normalized transcriptome library was produced using 454 pyrosequencing, assembled using Newbler and the genes predicted using ESTscan. Another transcriptome was produced using RNASeq data collected from tef seedlings subjected to various moisture regimes. The sequences were assembled using both Trinity and Oases/Nelvet and the coding regions predicted using ESTscan.

mate-pair Illumina and 454-FLX sequences were then used to link the contigs into scaffolds. GapCloser from SOAPdenovo was then used to close the gaps in the assembly. The assemblies were performed with k-mer values of $25,29,33,37,41,45$ and 49 , and the assembly with kmer value 41 was chosen based on assembly statistics and the presence of known sequences.

Approximately $80 \%$ of the tef genome was represented in scaffolds greater than $1000 \mathrm{bp}$. Around half of the tef genome was contained in the 3165 scaffolds that were aligned to sorghum following the requirement that at least three syntenic genes be identified. Re-sequencing the tef genome using advanced sequencing technologies with long reads such as Pacific Biosystems RS II technology and Illumina's Moleculo will contribute to the further improvement of the current assembly.

\section{Transcriptome sequencing and assembly}

A normalized transcriptome library prepared from roots and shoots of tef seedlings generated a total of $350 \mathrm{Mbp}$ of sequence reads using the 454-FLX technology (Additional file 2: Table S2). The reads were assembled with Newbler
[27] and resulted in a transcriptome (the '454Isotigs' transcriptome) with 27756 gene clusters and 38333 transcripts (Additional file 2: Table S3).

A second non-normalized library was obtained from various tef tissues subjected to drought and water-logging for the purpose of determining expression. It was then sequenced with the Illumina HighSeq 2000 to obtain 17 Gbp after trimming. The reads were assembled using Trinity [28] and Oases/Velvet [29]. These two assemblies were combined with the 454Isotigs and then clustered with TGICL, resulting in the 'Extended' transcriptome containing 28113 gene clusters and 88078 transcripts. Thus the two assemblies revealed substantial differences in the numbers of transcripts but similar numbers of gene clusters.

The large variation in the numbers of transcripts generated from the two assemblies stems from differences in the sequencing data and assembly procedures used. The 454Isotigs transcriptome was created from a normalized library using the Newbler assembler on long reads. On the other hand, the Extended database was constructed from merging three datasets, the 454Isotigs, 
a Trinity assembly made from short read data and an Oases/Velvet assembly made from short read data, a procedure which created much redundancy. To decrease the redundancy, the resulting transcripts were clustered with TGICL producing the 88,000 transcripts. Interestingly, this approach was also recently used by Nakasugi et al. [30] who concluded that it is advantageous to combine the output from many different de novo assemblers. A disadvantage is that this procedure increases the probability of chimeric sequences. The distribution of sequence lengths in the extended dataset has longer sequences than that of the 454Isotigs and is closer to the distribution of sequence lengths in sorghum and to the genomic predictions (Additional file 2: Figure S2). While 99\% of the 454Isotigs transcripts can be found in the Extended dataset, only $72 \%$ of the Extended can be found in the 454Isotigs (Additional file 2: Table S4). The number of clusters is remarkably similar between the two data sets and sorghum.

\section{Genome quality assessment}

Assembly statistics show that the quality of the tef genome is comparable to that of foxtail millet (Setaria italica) $[31,32]$ and other recently reported genomes [33-36] (Additional file 2: Table S5). The assembled tef genome has a size of $672 \mathrm{Mbp}$, equivalent to $87 \%$ of the estimated size of the sequenced genotype [13]. The distribution of k-mer frequencies was estimated with jellyfish [37] using $100 \mathrm{bp}$ reads from the $300 \mathrm{bp}$ insert-size library. K-mer statistics were used to estimate the genome size and varied from 650 to $700 \mathrm{Mbp}$ depending on the value of $\mathrm{k}$ (Additional file 2: Figure S3) [38,39]. The number of scaffolds greater than $1000 \mathrm{bp}$ in size was 14000 and the scaffold N50 was 85000 . The percentages of reads mapped to genomic scaffolds greater than 1000 bp in length are compared in Additional file 2: Table S6. Of the single reads, $84 \%$ could be mapped back onto scaffolds. For the 300 bp insert-size library, an average of $74 \%$ was mapped with the proper paired-end relationship.

To assess the quality of the genome, four sets of known tef sequences were sought in the genome. First, of the 496 pairs of primers reported for SSR genetic markers [8], 77\% were found in the current genome with a distance between them comparable to that expected (Additional file 3: Table S7). Second, as described in Additional file 2: Note 1 , two regions, one approximately $10 \mathrm{kbp}$ and the other approximately $8 \mathrm{kbp}$, were amplified, sequenced with the Sanger method and aligned to the genome. Percentage identities of 99 and $97 \%$, respectively, were obtained for the two constructs (Additional file 2: Table S8; S9). Third, from our previous efforts at genetic improvement, complete or partial sequences of several genes were made by Sanger sequencing. All of the genes and more than $92 \%$ of the bases in the genes totaling $31 \mathrm{kbp}$ were found in the draft genome (Additional file 2: Table S10; S11). Fourth, of the 3603 tef ESTs available in the NCBI database, approximately $99 \%$ were found. Therefore, the content of the draft genome is sufficient for the intended application to genetic improvement.

\section{Transcriptome quality assessment}

The distribution of lengths of proteins predicted from the Extended and the 454Isotigs transcriptomes were compared to that of sorghum (Additional file 2: Figure S2). While the distribution of protein lengths predicted from the 454Isotigs appears to be skewed toward shorter proteins, the distribution of protein lengths of the Extended dataset is similar to that of sorghum. In addition, the transcriptomes of the 454Isotigs and the Extended were compared to each other and to the sequenced sorghum genome [40]. The percentage of genes and bases aligned are tabulated in Additional file 2: Table S4. For each transcriptome, approximately $99 \%$ of the genes had at least a partial hit in the tef genome while $96-99 \%$ of the bases in the transcriptomes could be aligned to the genome. Over $90 \%$ of the sorghum genes had at least a partial match in the tef genome but many sequences were incomplete or substantially different as the number of bases of sorghum genes aligned to tef was less than $60 \%$. The 454Isotigs appeared to be a subset of the Extended transcriptome as almost all of the 454Isotigs were found in the Extended set while only $23 \%$ of the latter were present in the 454Isotigs.

\section{Comparison to other grasses}

Tef belongs to the grass family Poaceae which includes all cereal crops, and to the subfamily Chloridoideae and is the first sequenced member of this subfamily. The evolutionary relationships between the grasses are shown by the phylogenetic tree constructed from the waxy gene (Figure 2A). The closest cultivated species to tef is finger millet (Eleusine coracana), the genome of which is not yet sequenced. The closest subfamily to the Chloridoideae is the subfamily Panicoideae that includes sorghum (Sorghum bicolor) and maize (Zea mays). A phylogenetic tree constructed from orthologous genes between the five grasses which have been heretofore sequenced shows the evolutionary distances between the grasses (Figure 2B). Of the sequenced genomes, the closest to tef are foxtail millet (Setaria italica) and Sorghum bicolor.

The tef genome and the genes predicted in the genome from the Maker genome annotation pipeline combiner $[41,42]$ were uploaded to CoGe [43], a platform containing a wealth of genomes and providing interfaces to numerous tools for genome alignment, comparison, and visualization. CoGe's tool SynMap [44] identifies orthologous genes between two genomes using sequence homology and synteny. SynMap was used to identify syntenic tef scaffolds with at least five (alternatively three) genes having a 


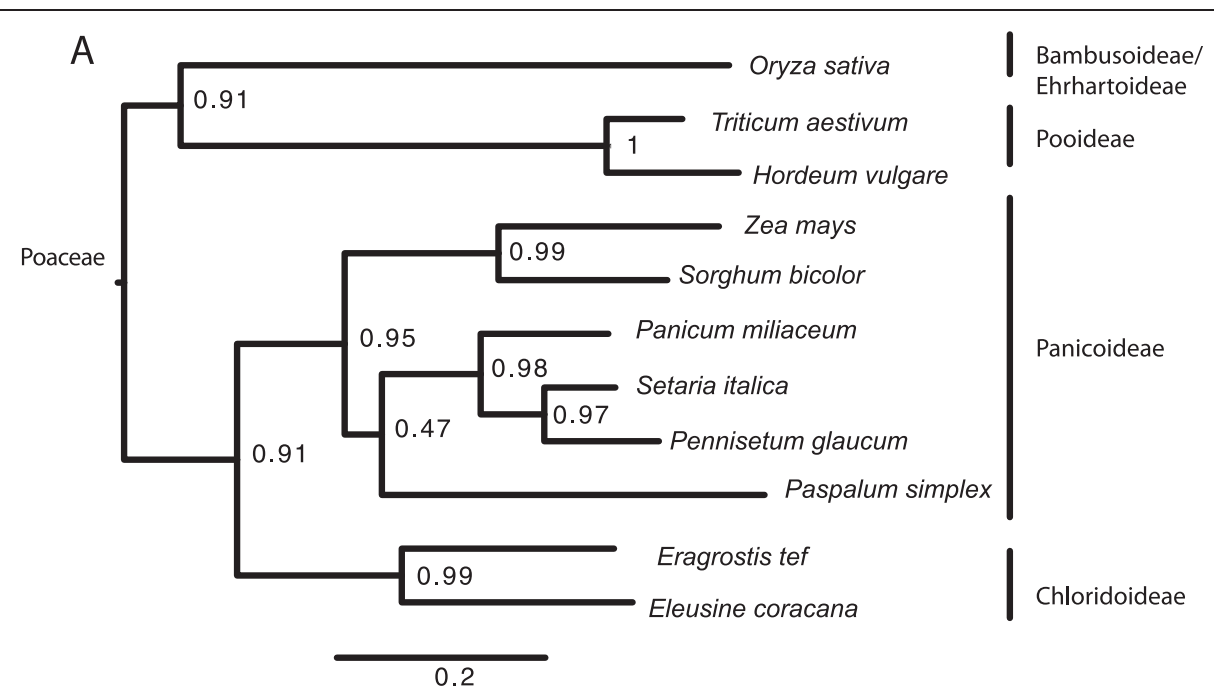

B

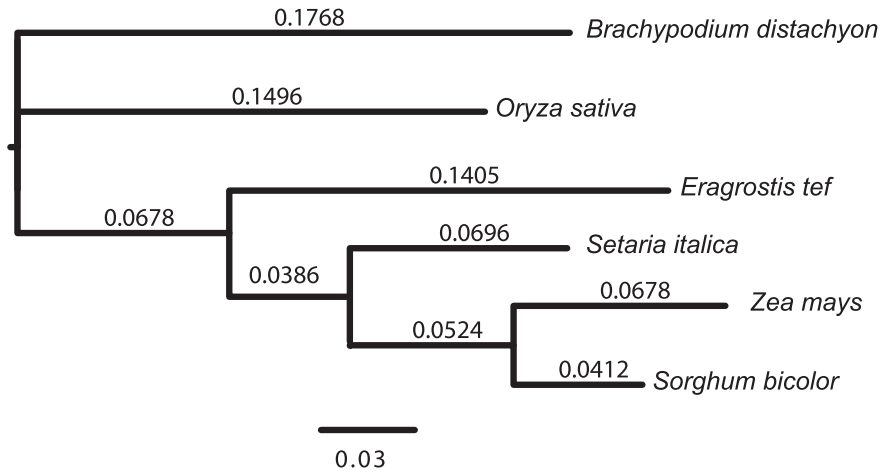

Figure 2 Phylogenetic tree for selected cereals from the grass (Poaceae) family including tef (Eragrostis tef). A) Partial sequences of the WAXY gene from barley (Hordeum vulgare, X07931), bread wheat (Triticum aestivum, KF861808), finger millet (Eleusine coracana, AY508652), foxtail millet (Setaria italica, AB089143), maize (Zea mays, EU041692), Paspalum simplex (AF318770), pearl millet (Pennisetum glaucum, AF488414), proso millet (Panicum miliaceum, GU199268), rice (Oryza sativa, FJ235770.1), sorghum (Sorghum bicolor, EF089839), and tef (Eragrostis tef, AY136939) were obtained from the NCBI database. The maximum likelihood tree was inferred using PhyML and the default model of HKY85 + G. The scale bar reflects evolutionary distance, measured in units of substitution per nucleotide site. Branch support was inferred using the Shimodaira-Hasegawalike (SH) aLRT provided by PhyML. B) Phylogenetic Tree of the Complete Grass Genomes. Protein supergenes with an aligned length of 260398 amino acids and constructed from orthologous sequences were used to infer a maximum-likelihood tree using PhyML with the WAG substitution matrix and a gamma model with four classes and an alpha parameter value estimated to be 0.489 . Branch lengths reflect the estimated number of amino acid substitutions per site. ML bootstrap values were all 100\%.

collinear genomic relationship to sorghum genes [45]. Alignment of these scaffolds to the 10 sorghum chromosomes produced 10 artificial tef 'pseudo-chromosomes'. A total of 2468 scaffolds containing approximately $346 \mathrm{Mbp}$ were ordered by this alignment of tef scaffolds to sorghum chromosomes (Figure 3A; Additional file 2: Table S5; Additional file 2: Note 2). These pseudo-chromosomes were constructed from a mixture of the A and $B$ genomes with the two homeologous scaffolds close to each other in the tef pseudo-chromosome. The scaffolds aligning to each sorghum chromosome were further sorted into A and B pseudo-chromosomes, by sequentially placing them into two groups based on overlap. The first scaffold was placed in $\mathrm{A}$, if the next scaffold had more than a $75 \%$ overlap with one in $\mathrm{A}$, it was put in $\mathrm{B}$. This was then repeated for all scaffolds in order, resulting in random placement of each scaffold into the A and B pseudo-chromosomes. Dotplots [46] of the A and B pseudo-chromosome show that they are homologous over large areas and also show the potential of syntenic mapping in sorting polyploid genome assemblies (Figure 3B; Additional file 4: Figure S4). A more sophisticated algorithm to identify which scaffolds belong to the $\mathrm{A}$ and $\mathrm{B}$ genomes is under investigation.

Genetic maps show the relative positions of loci with a distance based on the amount of recombination between them and are developed through markers which sort 


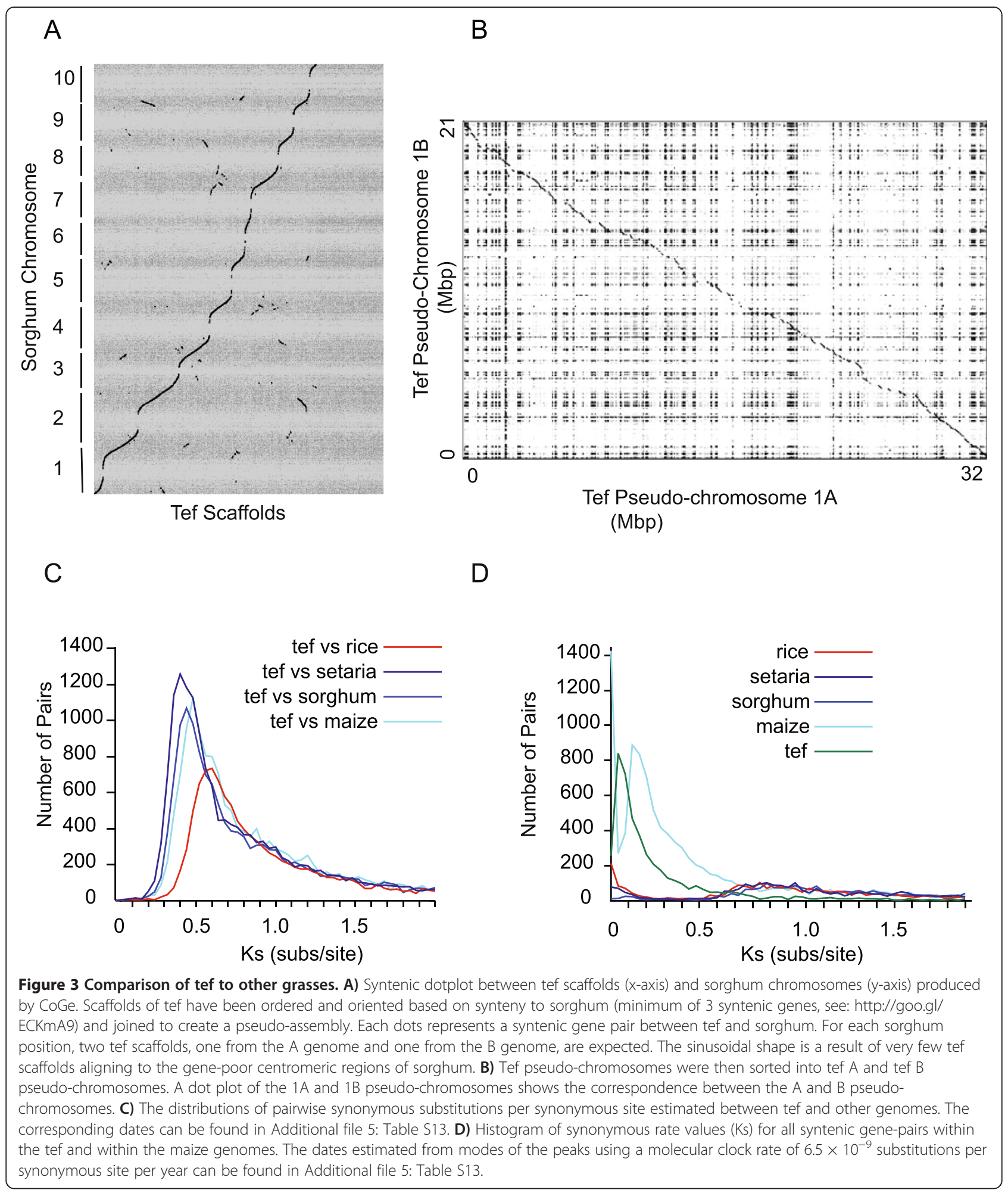

together based on phenotype. Physical maps give the distance between any two loci in units of base pairs. Establishing the relationship between the genetic and physical maps reveals the location of and distance between genes that are recombining together.
The available genetic map consists of 30 linkage groups instead of the 20 expected based on the chromosome number because there are not enough markers to join all of the linkage groups into chromosomes. By connecting the physical and genetic maps, we can establish which 
linkage groups correspond to each chromosome and which linkage groups are homeologous. The tef genome is not assembled into the 20 chromosomes so the pseudo-chromosomes created by mapping to sorghum were aligned to the genetic map of tef and used to suggest a putative order of the linkage groups [47] (Figure 4; Additional file 2: Table S12). For example, it can be seen that linkage groups 23,18 and 21 all map to tef pseudochromosome number 4 (dark green lines) while linkage groups 1, 2 and 3 all map to pseudo-chromosome number 3 . These could either be homeologous linkage groups or linkage groups that did not have enough markers recombining together to be joined.

Inversions and translocations between the tef and sorghum genomes could be seen such as the chromosomal translocation between tef linkage group 3 and tef pseudo-chromosomes 3 and 9 as well as an inversion between linkage group 3 and 5 that correspond to

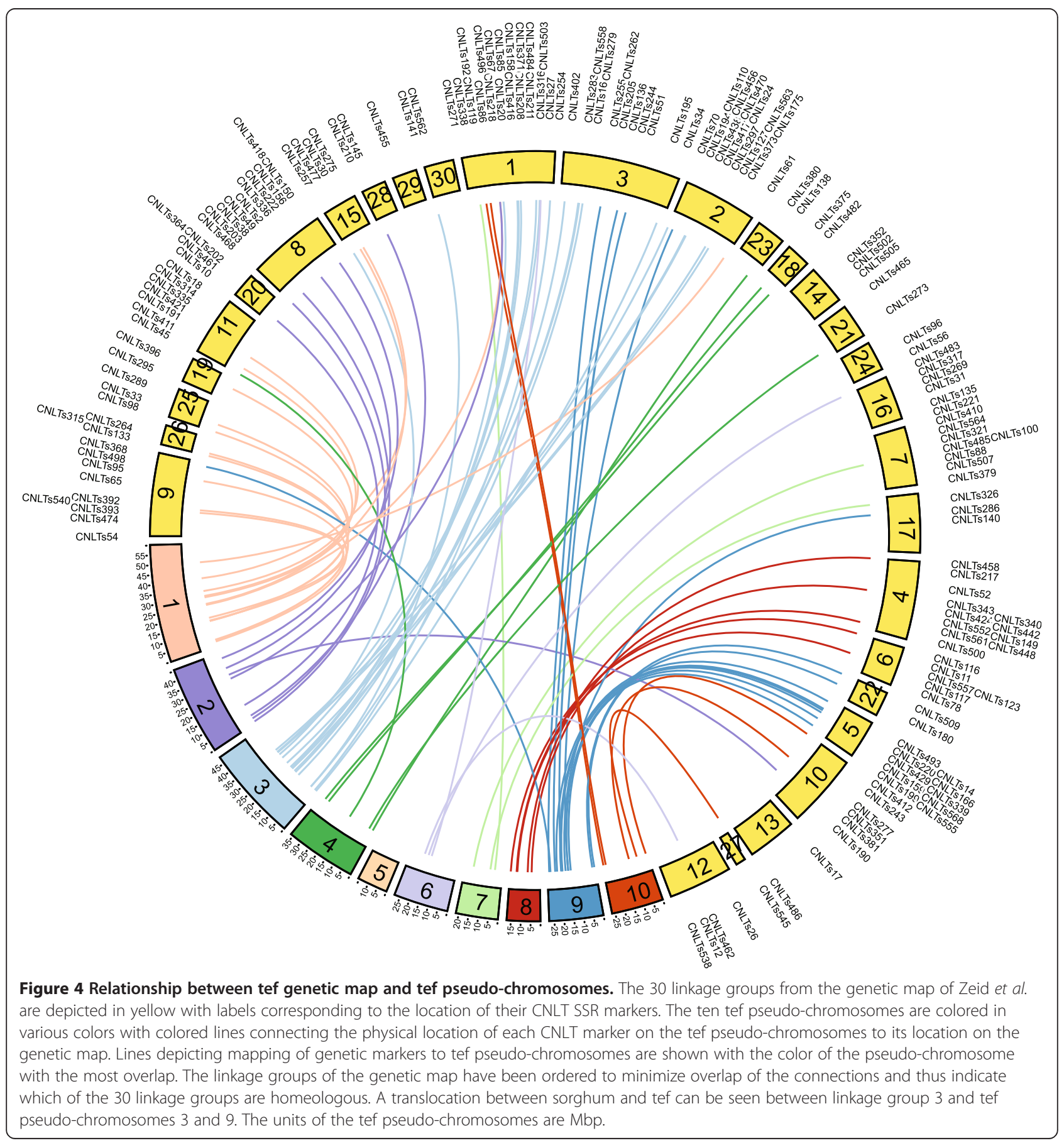


pseudo-chromosome 9. The physical distance and genetic distance were compared for 33 marker pairs and ranged between 0.003 and $15 \mathrm{cM} / \mathrm{Mbp}$ (Additional file 2: Table S12). The variation in the estimates might be related to the sparsely covered genetic map or because of differences in tef recombination rates.

Identification of the set of orthologous genes between two genomes enabled genome-wide comparisons. Codeml of PAML [48] is integrated into CoGe. Codeml is a program for the reconstruction of ancestral sequences using a collection of codon models which in turn allows estimation of synonymous (Ks) and non-synonymous (Ka) substitution rates over trees, branches or sites. The ratio of Ka to Ks is useful for the detection of selection in proteincoding DNA sequences while Ks alone can be used as a molecular clock. CoGe first identifies orthologous genes based on collinearity and then returns Ka and Ks values for the complete set of orthologous genes between two genomes. The Ks distribution between two genomes can be used to estimate the divergence date between the two species; the mode(s) of the distribution represents either a speciation or duplication event. The divergence date corresponding to the mode can then be estimated using a substitution rate, for plants a substitution rate of $6.5 \times 10^{-9}$ synonymous substitutions per synonymous site per year has been proposed [49].

The SynMap function of CoGe was used to do pairwise comparisons of the following genomes: Eragrostis tef (Coge id 38364), Sorghum bicolor (Coge id 38364), Zea mays (Coge id 333, B73 refgen_v2 assembly, working gene set annotations 5a), Oryza sativa japonica (Coge id 3) and Setaria italica (Coge id 32546, with CNS PL2.0 l v2.1, id2240) (Figure 3C; Additional file 5: Table S13). From the mode of the distribution at 0.47 substitutions per site, the divergence date between sorghum and tef was estimated to be around 36 million years ago (MYA). All genome-wise estimates of Ks between tef and other Poaceae are in Additional file 5: Table S13 and are comparable to those found by Smith et al. [10].

\section{Allotetraploidy}

Tef is an allotetraploid, the result of genome duplication by hybridization between two diploid progenitors. The whole genome duplication of tef is relatively recent in the history of the grasses and therefore it provides a unique snapshot into the consequences of such an event at this time point.

Whole genome duplications in grasses can be revealed by the distribution of Ks values within a genome. If a genome has undergone a recent whole genome duplication, the distribution of Ks values computed between the genome and itself will show a peak whose Ks value is an estimate of the evolutionary distance between the homeologs.
In order to determine the evolutionary distance between the two sub-genomes in the grasses, the distribution of Ks values between homeologous genes within each genome was computed using CoGe. Maize and tef had evident peaks corresponding to recent whole genome duplications while the other grasses did not (Figure 3D). The mode of the tef Ks distribution was 0.05 substitutions per codon, while that of the maize distribution was 0.15 , corresponding to genome duplications for tef and maize occurring approximately 4 and 12 MYA, respectively, using a substitution rate of $6.5 \times 10^{-9}$ synonymous substitutions per synonymous site per year [49] (Additional file 5: Table S13). These divergence dates are in agreement with previous estimates for tef [10] and maize [50]. A small broad peak centered around 0.9 substitutions per site is present in all genomes and corresponds to the rho whole genome duplication, a duplication that occurred prior to the divergence of the grasses [51].

Alignment of the tef A and B pseudo-chromosomes showed that the average sequence identity between the aligned nucleotides of the A and B pseudo-chromosomes over the entire genome was $93 \%$ not counting the frequent indels (Additional file 2: Table S14). Eight genomic sequences for which Sanger sequences were available for both homeologous copies had an average of $96 \%$ sequence identity in the coding region and $82 \%$ in the non-coding region (Additional file 2: Table S15).

In addition, as there are two homoeologs expected for each gene in the genome, two copies of each transcript of the 454Isotigs and the Extended transcriptomes were sought in the genome. Statistics were tabulated for longest aligning copy, 'copy 1 ' and the second-longest aligning copy, 'copy 2' (Additional file 2: Table S16). For the 454Isotigs, $96 \%$ of the transcripts were found and $86 \%$ of the transcripts had a second copy, indicating that the majority of the genes are present with two copies.

\section{Discovery of novel SSR markers}

Simple Sequence Repeats (SSRs) or microsatellites are sequences of 2-6 nucleotides that repeat from 3 to 100 times. They are highly polymorphic and therefore often used as molecular markers for breeding indigenous crops such as tef. Although high-throughput sequencing of SNPs for genetic markers is becoming more commonplace, SSR markers are still important for breeding in the developing world. Marker-assisted breeding depends on the natural variation in the population. Of the 496 pairs of primers reported for SSR markers in tef [8], only $77 \%$ were found in the current genome mainly due to its source. The SSR markers were developed from ESTs which represent only the transcribed regions while the genome sequence contains both transcribed and non-transcribed regions. Hence, the primer sequence of an SSR marker might be split into two places in the 
genome due to the presence of an intron. These divided markers would not be detected in the genome. Markers could also be missing from the genome either because they were not sequenced or because of natural variation between the different tef cultivars used for sequencing.

About 49600 SSR repeats (di-nucleotide or larger) or about one every 13565 bases were found in the tef genome (Additional file 2: Table S17) using MISA [52]. MISA is a tool developed to systematically search for SSRmotifs. Perl scripts provided on the MISA website to interface MISA with Primer3 [53,54] were used to design primers for all SSRs with repeat size of three or more (Additional file 6: Table S18 for the entire genome). Several SSRs having a close proximity to a gene cluster controlling yield or drought on the genetic map [47] but not in coding regions were chosen for PCR amplification. To maximize the chance of polymorphisms, the SSRs were chosen to be in noncoding regions.

A novel SSR located near known markers on linkage group 9 was found to have differences between the Alba and Tsedey cultivars of tef, and was further investigated (Additional file 2: Figure S5A; Additional file 2: Table S19). Approximately 200 bp surrounding the SSR was sequenced for 20 tef cultivars (of which Tsedey, Dukem, Magna and Quncho are improved varieties) as well as four wild Eragrostis species (Additional file 2: Figure S5B). The genotypes were variable at 32 positions including indels. Addisie, Beten, Gommadie, Kaye Murri, Magna, Rosea, Tsedey and Variegata shared the same number of repeats of the CTCCT motif while Ada, Alba, Balami, Dabbi, Dukem, Enatite, Gea Lammie, Karadebi, Manyi, Quncho, Red Dabbi and Tullu Nasy, had one less (Additional file 2: Figure S6). In addition, Tullu Nasy, Alba and Balami share an indel. Of the wild species, $E$. pilosa and $E$. minor had two fewer instances of the repeat while E. curvula and E. trichodes had three fewer occurrences. The sequence alignment and the most parsimonious phylogenetic tree based on all variable positions support these relationships and place the Gommadie and Kaye Murri ecotypes, which both have a semi-compact panicle, to the same group (Additional file 2: Figure S7). A parsimony tree with the same score could be obtained by putting E. minor in the same clade as E. pilosa A to the exclusion of E. pilosa B.

Discovery of a novel SSR marker indicates that the genome has applications in marker-assisted breeding. This new marker was located on scaffold2788 at position 199935 only $90 \mathrm{kbp}$ from the rht1 (reduced height) gene responsible for semi-dwarf stature in wheat and rice [55]. It separates 20 tef genotypes with diverse agronomic and morphological traits [56] and four closely related wild Eragrostis species into two groups, while other polymorphisms in the surrounding sequence further divide the groups. The phylogenetic relationship between several natural accessions as well as two improved varieties could be determined from sequencing the locus around the SSR. Among the four improved varieties, two (Magna or DZ-01-196 and Dukem) were developed via widely practiced selection procedures while the other two (Quncho and Tsedey) were developed through introgressions between improved tef cultivars. A cross between the high-yielding Dukem variety and the white-seeded Magna variety produced Quncho, the most popular variety in Ethiopia [57]. For the region sequenced here, the Quncho sequence is identical to that of the parent Dukem and not the parent Magna. Although most of the polymorphisms occur between the different Eragrostis species, the tef natural accessions Kaye Murri and Ada also have point mutations.

\section{Genome annotation and analysis}

Genes were predicted in the genome using the Maker evidence combiner $[41,42]$. Over $92 \%$ of the predicted genes were supported by the Extended transcriptome. The distribution of gene lengths of the predicted genes was consistent with that of the sorghum genome (Additional file 2: Figure S2). Annotation was performed with the Swiss Institute of Bioinformatic's Praise Annotation System, designed to use manually curated rules to combine annotation from different sources (Additional file 2: Note 3). The numbers of entries with various annotations for the different datasets can be found in Additional file 2: Table S20.

Using WindowMasker with the default settings, 14\% of the genome was masked (Table 1) which is substantially lower than that expected from other grasses such as sorghum with $\sim 61 \%$ [40]. RepeatMasker using the TREP database [58] as input found only $6 \%$ of the draft genome to be transposable elements, $3.9 \%$ retroelements (class I transposable elements) and 2\% DNA transposons (class II transposable elements). The number of repeat elements found was small but given the genome assembly procedure used, it is expected that the number of repeats is greatly reduced due to collapsed sequence assemblies [59]. Infernal [60] with the Rfam database [61,62] identified 80 rRNAs (99 in foxtail millet), 1184 tRNAs (577 in sorghum, 704 in foxtail millet, 1163 in maize), 570 miRNAs (159 in foxtail millet) and 834 snRNAs (382 in foxtail millet) $[32,63]$.

Miniature inverted-repeat transposable elements (MITEs) are a special type of class II non-autonomous transposable elements (TE) that are abundant in the non-coding regions of the genes of many plant and animal species [64]. The number of MITEs found in the tef genome is 77908 which is $1.6 \%$ of the genome size (Table 1). Around 56000 MITEs were identified in sorghum, comprising $1.7 \%$ of the genome [40] while Oki et al. [65] reported 73500 MITEs in rice, comprising $5.2 \%$ of the genome. 
Table 1 Summary of genome annotation

\begin{tabular}{|c|c|c|c|}
\hline Type & Number or copies & Total size (bp) & Percent of genome \\
\hline \multicolumn{4}{|l|}{ A. Repetitive Elements } \\
\hline Masked with WindowMasker & & 95556652 & 14.2 \\
\hline Masked with RepeatMasker & & 39352657 & 6.6 \\
\hline MITES & 77908 & 10783061 & 1.6 \\
\hline \multicolumn{4}{|l|}{ B. Noncoding RNAs } \\
\hline rRNA & 80 & 13580 & \\
\hline tRNA & 1184 & 88295 & \\
\hline miRNA & 570 & 73999 & \\
\hline snRNA & 834 & 95690 & \\
\hline
\end{tabular}

\section{Proteins}

$\begin{array}{lcccc}\text { Dataset } & \text { Number of clusters (unigene) } & \text { Number of transcripts } & \text { Average length of CDS (bp) } & \text { Percentage of Genome } \\ 454 \text { Isotig } & 27756 & 38333 & 285 & 1.8 \\ \text { Extended } & 28113 & 88078 & 331 & 4.7 \\ \text { Maker prediction } & & 42052 & 395 & 3.3\end{array}$

Number and size of A) repetitive elements, B) noncoding RNAs, and C) proteins. Repetitive elements were quantified using WindowMasker, RepeatMasker and MITE-Hunter. The non-coding RNAs were found using the Infernal software with the Rfam database. The transcripts in two assemblies (454lsotigs, Extended) and the predicted genes in the genome (Maker) are also compared.

\section{Abiotic stress related genes in tef}

Although tef is relatively drought tolerant, moisture scarcity is among the major yield limiting factors in tef production [66] and severe drought remains a critical problem for Ethiopia [67]. One of the goals of the Tef Improvement Project is to identify genes involved in tef's abiotic stress tolerance, in particular those for drought-tolerance, and to use them to develop more drought-tolerant cultivars. Tolerances to abiotic stresses such as tolerance to drought, salt, and water-logging are complex traits controlled by many genes. Access to the genomic sequence of tef enables the transfer of knowledge of these genes gained from wellstudied crops like maize and rice to the application of molecular-supported breeding efforts in tef. In addition, the tef genome provides a new pool of abiotic stress target genes.

Twenty-six Arabidopsis, rice and sorghum genes known to be involved in abiotic stress response were investigated by aligning each query sequence to the tef genomic sequences (Additional file 2: Table S21). Two homeologs were sought in tef. For comparison, they were also aligned to the sorghum and foxtail millet genomes. All of the genes were found at least partially in all genomes. The percentage of query sequence nucleotides aligned to the tef genome differed for each gene and ranged from 57 to $100 \%$. These percentages were similar to those found in foxtail millet. The percentages of the query sequences aligned to sorghum were higher than both tef and foxtail millet, not surprising given that some of the query sequences were from sorghum and that the quality of the sorghum genome is higher. For 21 of the 26 genes investigated, two homeologs were found in tef.
One of the goals of this project is to identify molecular breeding targets. To investigate which genes might have special adaptations in tef, gene families that have undergone expansion or contraction were sought as this might provide some clues to the adaptation of tef to extreme climatic conditions in general and specifically to drought tolerance. For this reason, the number of family members for each of these genes was counted in tef and the other grasses and tabulated (Additional file 2: Note 4; Table S22).

Among these genes known to be involved in drought tolerance in several plant species, we have singled out the SAL1 gene which is involved in abiotic stress tolerance in both monocts and dicots. Mutants with downregulated or inactivated SAL1 genes have been found to have an increased drought tolerance in Arabidopsis [68] and wheat [69]. From Additional file 2: Table S22, SAL1 was also identified as a family in which gene duplication may play a role in the drought response of tef. For these reasons, SAL1 was chosen for detailed analysis.

Family members of the SAL1 gene were identified by using homology searching of the sorghum SAL1 gene in the genomes of five grasses. Seven copies or partial copies of the SAL1 gene were found in tef compared to 1-2 in the other grasses investigated (sorghum, foxtail millet, Brachypodium and rice). The phylogenetic relationship of the members of the SAL1 family is shown in Figure 5A. Two gene subfamilies are present, probably the result of a tandem duplication which took place before the divergence of the grasses. Both copies have been retained by all grasses with the exception of Brachypodium for which only one copy was found. For tef, in addition to a second 
A

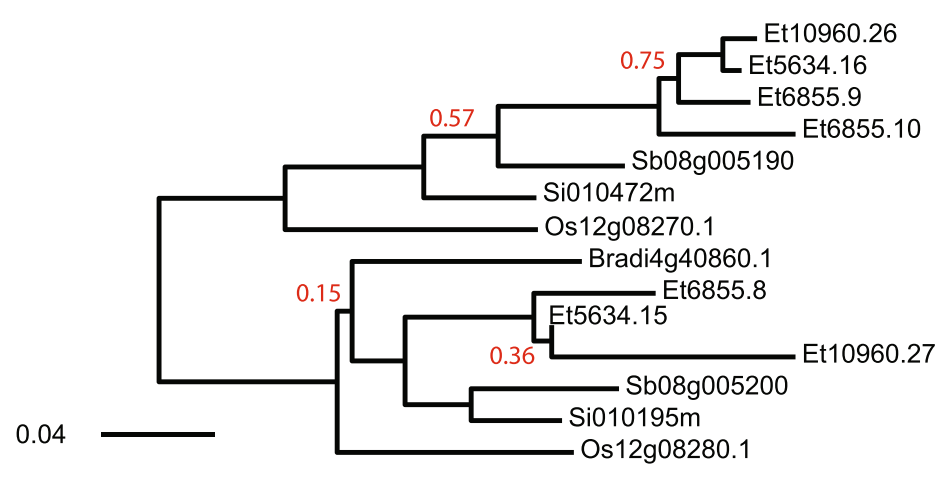

B
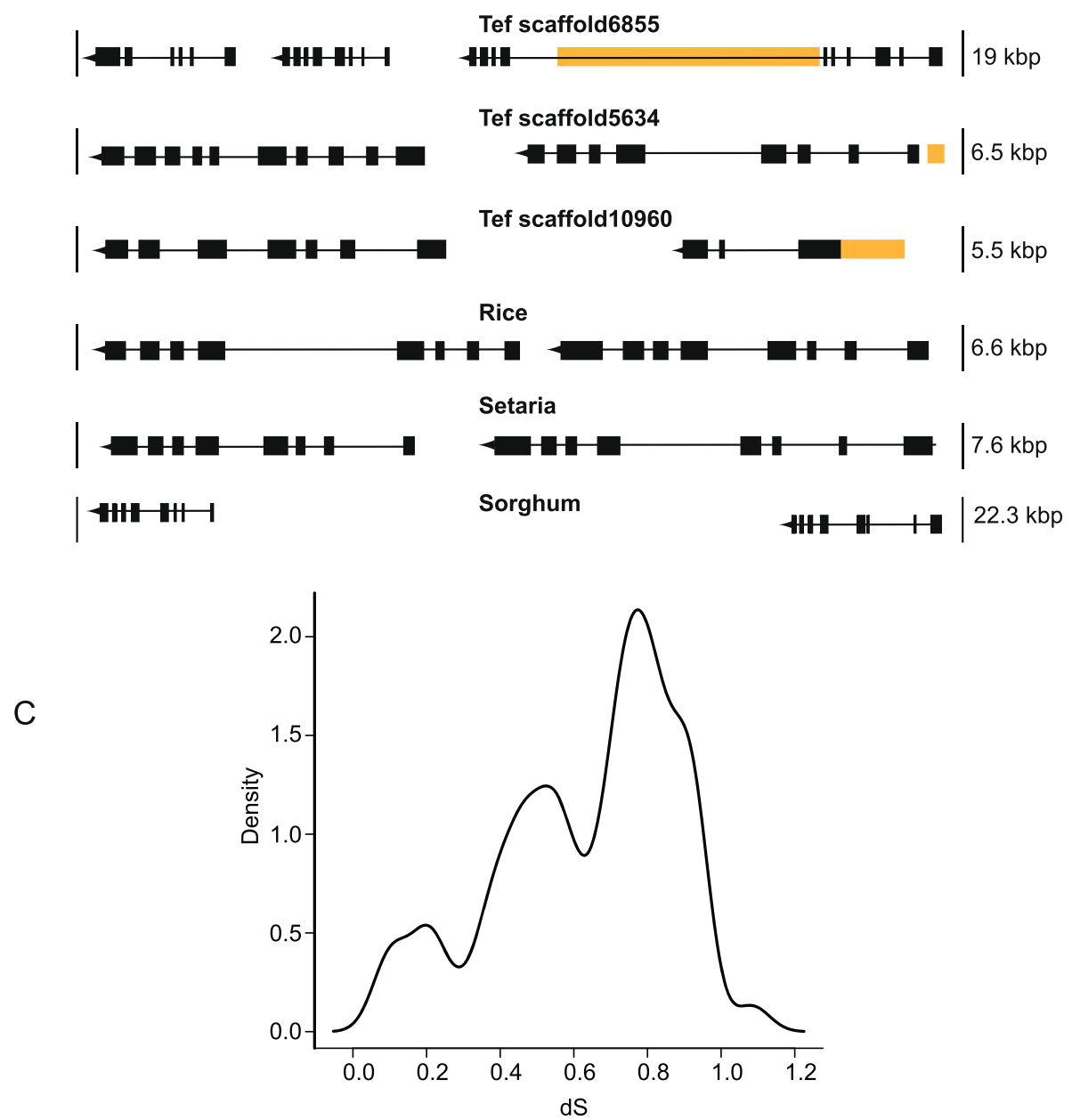

Figure 5 SAL1 gene in tef and other grasses. A) The SAL1 gene duplicated before the divergence of the grasses and has also undergone several recent duplications in one branch of the phylogenetic tree. The ML tree was constructed using the WAG protein substitution model implemented in PhyML (version 3.0). Branch support was inferred using the conservative and non-parametric Shimodaira-Hasegawa-like (SH) aLRT provided by PhyML. Only branch support values less than 0.85 are shown. Abbreviations: Et: Eragrostis tef; Sb: Sorghum bicolor; Os: Oryza sativa; Bradi: Brachypodium distachyon; Si: Setaria italica. B) Comparison of orthologous syntenic genomic regions between tef, sorghum, and setaria. The SAL1 gene appears on three scaffolds in the tef genome, twice as tandem duplicates and once as a tandem triplicate and is found as a tandem duplicate in rice, setaria and sorghum. Orange blocks indicate unsequenced regions created from the scaffolding. C) Distribution of Ks values for all pairwise comparisons of SAL1 gene family members. 
homologous copy, it appears that the entire region has been duplicated yet another time as this sequence of genes appears in three scaffolds in its entirety (scaffold6855, scaffold5634, scaffold10960). In addition, on scaffold6855, SAL1 appears as a tandem triplicate (Figure 5B) indicating further gene duplication in this family. The presence of this particular tandem triplication was confirmed by designing several sets of PCR primers in the region spanning $12.5 \mathrm{~kb}$ and sequencing the PCR products using the Sanger method. As shown in Additional file 2: Figure S8, all three SAL1 tandem genes identified in the scaffold are present in the tef genome.

Analysis of $\mathrm{dN} / \mathrm{dS}$ values identified no branches of the tree evolving under positive selection. The distribution of $\mathrm{dS}$ values between the members of the SAL1 family examined shows a peak at 0.8 substitutions per codon which reflects the distance between the two subfamilies that have been diverging since the original duplication (Figure $5 \mathrm{C}$ ). A peak around 0.5 substitutions per codon reflects the speciation between the most distant members of each subfamily. The smallest $\mathrm{dS}$ value of 0.1 was located between Et10960.26 and Et5634 indicating either a recent duplication or gene conversion. The dS value between Et6855.9 and Et6855.10 indicates that this is the most recent duplication of the triplication on scaffold6855.

In addition to the SAL1 gene, several other genes known to be involved in abiotic stress tolerance have been identified as potential targets for improvement by observing changes in the number of family members both in the genome and transcriptome constructed separately from the control, drought and waterlogged RNASeq samples. These included B-glucanase [70], SGR [71] and ERD1 [72], all involved in the response to drought (Additional file 2: Table S22). These potential candidates will be the next targets investigated in the laboratory.

\section{Prolamin family genes related to gluten sensitivity}

Celiac disease in humans is caused by an immune response to specific amino acid sequences, called epitopes, that are present in wheat, barley and rye gluten. The family of genes responsible is the prolamin gene family [5]. Antibody-based assays have shown that tef does not contain the offending epitopes [7]. Possession of the genomic sequence allows for confirmation of these assays. A comprehensive search of all wheat, barley and rye epitopes causing immune reactions in celiac patients resulted in 96 epitopes that stimulate T-cells [5]. These 11-, 12-, 13-, 16and 20- amino acid-long epitopes were sought in tef, Brachypodium, barley, rice, maize, Setaria and sorghum genomes as well as the wheat and rye sequences at NCBI. None of these epitopes were found outside of the wheat, barley or rye genome (Additional file 2: Note 5; Table S23) confirming that rice, maize, sorghum and tef products may be safely consumed by celiac patients.
Prolamin genes were sought in the tef genome by using BLAST [73] to search for homologs to grass prolamin protein sequences described by $\mathrm{Xu}$ and Messing [74]. In the tef genome, 23 sequences were found (Figure 6; Additional file 2: Table S24), often as tandem duplicates, with the majority being located on tef pseudo-chromosomes 3, 7, 9 and 10. Many of the genes were found to have stop codons or frame shifts. Two were expressed either in the transcriptome or in mass spectrometry analysis of tef [75].

As detailed in $\mathrm{Xu}$ and Messing [74] grass prolamins fall into three groups. Group one includes alpha- and delta- prolamins. Alpha prolamins are present in the Panicoideae (millet, maize, sorghum) but not rice or Pooideae (wheat, barley, Brachypodium). They are the youngest family of prolamins and thought to have originated from delta prolamins. One copy of tef alpha prolamin was found in both the genome (scaffold10996) and the transcriptome (isotig15824), indicating that it is being expressed (Figure 6; Additional file 2: Table S24). The majority of the genomic copies found in the tef genome are delta prolamins including one expressed in our transcriptome studies (CL17177Contig1). The delta group includes a tandem triplication on scaffold2167 as well as a tandem duplication on scaffold 7847.

Group two of the prolamins, the largest group, contains gamma and beta zeins/kefarins in maize and sorghum as well as the S-rich prolamins (alpha- and gamma-gliadins, gamma- and beta-hordein, gamma secalin, and the LMW prolamins in wheat, barley and rye). One genomic copy of a beta-like tef sequence and one genomic copy of a gamma-like tef sequence were found. For this group, the Pooideae cluster together as do sorghum, maize and tef.

Group three, comprised of prolamins only found in Pooideae, is more closely related to alpha-globulins (nonprolamins) than to other prolamins. Tandem duplicates of two alpha-globulin genes were found on scaffold4989 and scaffold4451, both located on tef pseudo-chromosome 9.

The only tef prolamin sequences found in the literature were produced by Tatham et al. [75] who identified and isolated tef prolamin sequences using SDS-PAGE and HPLC. Six peaks were resolved with HPLC, for two of them (tef 2 and tef6), the first 30 amino acids were sequenced. They concluded that both tef6 and tef2 were alpha prolamins based on the high similarity of their amino acid compositions to the amino acid composition of the alpha-prolamins of maize. The sequence of tef6, similar to scaffold10966, is found in the transcriptome as isotig15824 and appears to be an alpha prolamin. The 30 amino acids of Tatham's peptide tef 2 were found several times in the tef genome; the locations are tabulated in Additional file 2: Table S24. However, no prolamin genes were found in these regions when searching for homology with members of the prolamin family. Therefore the full sequence and status of tef2 remain 


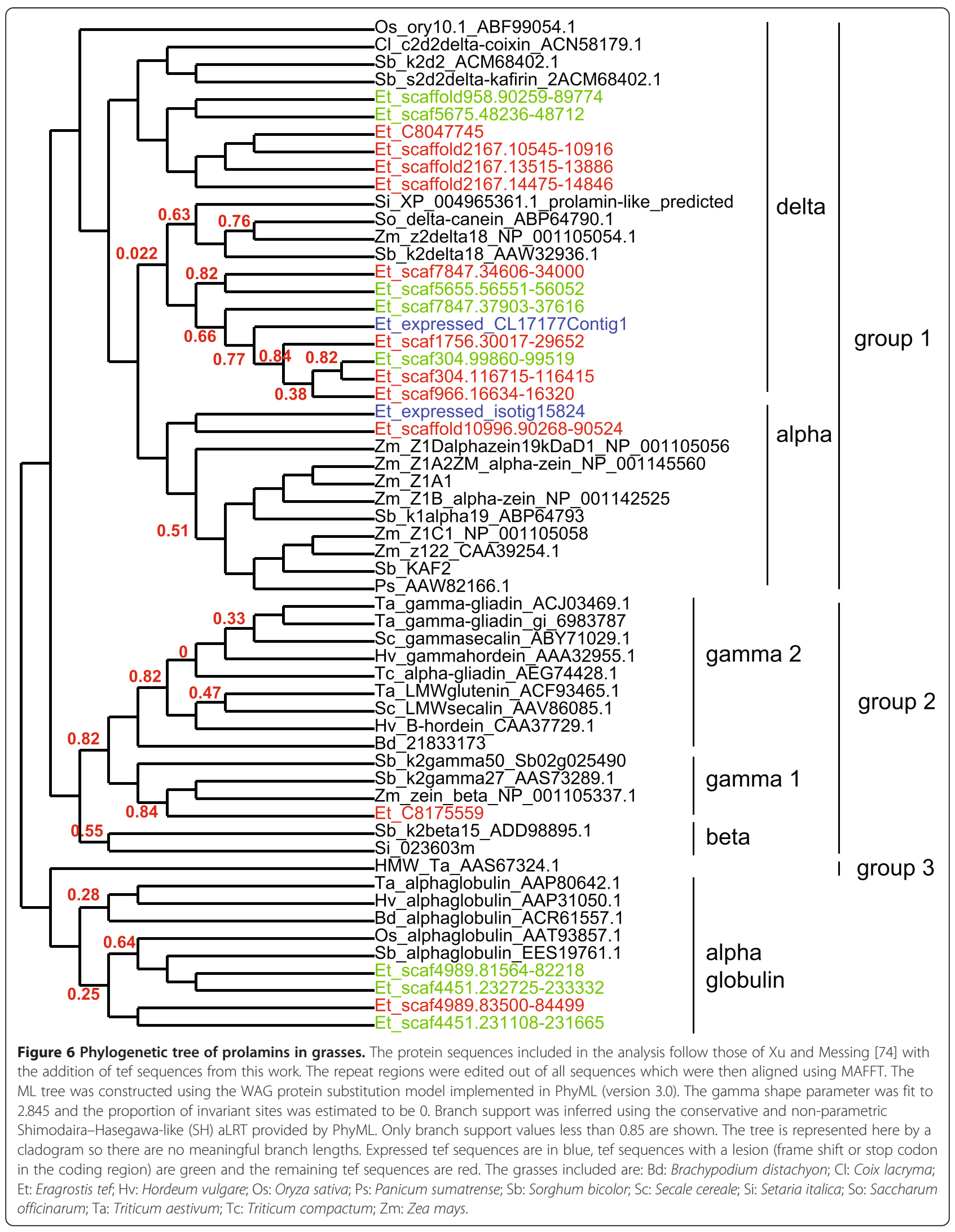


unknown. The 30 amino acids of tef2 were also not found in the transcriptome.

Both the tef and the finger millet sequences had Nterminal deletions compared to zeins as is the case with many of the tef sequences found in this analysis. Surprising is the large amount of duplication that has occurred in the delta prolamins of tef. Seed tissue was not included in the sequencing library; therefore, it is uncertain if all of the expressed genes have been detected.

\section{Conclusions}

The genome sequence of tef, an indigenous and economically important cereal crop in the Horn of Africa, is a valuable resource for comparative and functional genomic studies of grasses, particularly for abiotic stress tolerance and healthy nutrition for which tef can be considered as model plant. In general, the genome of tef provides a molecular basis for improvement techniques that will provide new cultivars for subsistence farmers in Ethiopia. Furthermore, the genome sequence of tef is a starting point for the exploration of the genetic diversity in tef natural accessions and mutagenized populations. It paves the way for the application of modern techniques such as EcoTILLING, Genome Wide Association Studies (GWAS), Genotyping by Sequencing (GBS) and RNASeq in harnessing the rich natural variation present in the tef germplasm.

\section{Methods}

\section{DNA sample preparation}

Genomic DNA was extracted from two-week-old Tsedey (DZ-Cr-37) seedlings using the Nucleospin Plant II maxi kit (Macherey-Nagel) according to the supplier's protocol. The DNA quantity and quality were measured using an ND-1000 Spectrophotometer (Nano-Drop, USA) in which the ratio of $260 / 280$ wavelengths was 1.87 .

\section{Genome sequencing}

Illumina sequencing reads were collected from a HiSeq2000 Machine from Fasteris, Geneva [76]. 454 reads were sequenced using the 454-FLX technology from the Functional Genomic Center, Zürich, Switzerland [27,77] and Macrogen, Korea [78] according to standard protocols. For HiSeq2000 sequencing, various library preparations and sequencing protocols were investigated and the best results obtained using the Accuprime polymerase (Invitrogen, Carlsbad, CA) and following the protocol for high GC content.

\section{Genome assembly}

FastQC [79] was used for quality control. Sequence pairs containing adaptor or primer sequences were removed and the sequences were trimmed such that all positions had a Phred score greater than 28. All Illumina and the single-end 454-FLX sequences were assembled into contigs using SOAPdenovo [80] with parameters ' $-\mathrm{L} 100,-\mathrm{R}$ yes, max_rd_len $=85$ '. The paired-end and mate-pair Illumina and 454-FLX sequences with insert sizes of $3 \mathrm{~kb}, 4 \mathrm{~kb}, 6 \mathrm{~kb}$ and $13 \mathrm{~kb}$ were then used to link the contigs into scaffolds. GapCloser was used with parameter '-p 31'.

\section{Repeat analysis}

WindowMasker [81] with its default settings and the '-dust' option was used to identify repetitive sequences based on n-mer frequency counts in the genome. The '-dust' option identifies and masks regions of low complexity regions in addition to interspersed repeats. In addition, repeats were masked using RepeatMasker [82] with the TREP nucleotide and protein databases of plant repetitive elements [58]. MITE-Hunter [64] with the default parameters was used to find MITEs.

\section{Gene prediction in the genomic sequence}

Gene predictions were performed using the evidence combiner, Maker, on the unmasked genome using Augustus predictions [83] with the maize matrix. The Trinity transcriptome before clustering was used as EST evidence and the complete Uniprot Swissprot database from September 2012 was used as protein homology evidence. For repetitive sequence finding, all model organisms were used from RepBase, the TREP11_beta database was used as the organism specific repeat library and the te_proteins.fasta file provided with Maker was also used. After the first iteration, SNAP [84] was trained on the Maker output from the first run and then Maker was rerun. Two such iterations were performed.

For identification of tRNA genes, tRNAscanSE version 1.3.1 was used [85]. All non-coding RNAs [60] (including tRNAs) were identified using the perl control script rfam_scan.pl version 1.0.4 with Infernal version 1.0 and the Rfam database version 11.0. In addition tRNAscan version 1.3.1 was used. A database of tef MITEs was created using MITEHunter version 11-2011 with the default parameters [64]. This database was then used in 'RepeatMasker version 3.3.0 with options' -nolow -no_is to find the complete set of MITEs in the tef genome.

\section{RNA sample preparation and sequencing for the normalized library}

All RNA was extracted from the improved tef variety Tsedey (DZ-Cr-37). To construct the normalized library, leaves from two-month-old plants, roots from ten-day-old seedlings grown in vitro, and seedlings exposed for three weeks to three conditions, namely waterlogging, drought, and normal watering, were harvested. The RNA was extracted from individual samples using the RNeasy kit (Qiagen, Switzerland) according to the supplier's protocol. The 
quality and quantity of RNA was quantified using ND1000 Spectrophotometer whereby the average 260/280 ratio was 2.0 indicating good quality RNA. Five $\mu \mathrm{g}$ of RNA from each of the above five samples was pooled. Library construction and sequencing was provided by MWG (Germany). A total of one million single-end reads (equivalent to $351 \mathrm{Mbp}$ ) with a mode of $400 \mathrm{bp}$ were collected. The data was filtered for primer and adaptor sequences and trimmed such that all positions had a Phred score greater than 28 .

\section{Transcriptome library construction and sequencing for RNASeq}

The RNA extracted from plants grown under waterlogging, drought, and normal watering conditions as described above, were sent to Fasteris (Geneva, Switzerland) for further quality testing and sequencing using Illumina HiSeq2000 with the intention of analysis for differential expression. From each of the three samples, two different libraries were prepared. Six cDNA libraries were sequenced to generate a total of 205 million single-end reads as shown in Additional file 2: Table S2. Before assembly, the reads were trimmed such that the Phred quality scores were above 28. In addition, all primer and adaptor sequences detected by FastQC were removed.

\section{Transcriptome sequencing and assembly}

Two transcriptomes (one from the normalized library '454Isotigs' and an 'Extended' set) were assembled. The 454-FLX sequences together with 3608 tef EST sequences (equivalent to about $1.8 \mathrm{Mbp}$ ) downloaded from NCBI were assembled with Newbler 2.3 [27], resulting in an assembly referred to as '454Isotigs' and having 27756 Isogroups and 38437 Isotigs. Prediction of coding regions using ESTScan 'with $\mathrm{m}=100, \mathrm{~N}=0, \mathrm{w}=60$, and using the Zea mays scoring matrix' resulted in 33098 predicted genes.

The RNASeq data from Illumina was assembled into two datasets, namely Illumina-Trinity and Illumina-Oases/ Velvet.

\section{Illumina-trinity}

The nine Illumina RNASeq data sets were assembled with Trinity [28] 2012-01-25p1 using the trinity.pl wrapper and options '-bflyHeapSpace 15G' and '-no_meryl'. In addition, the GNY1/GNY10 (control), GNY2/GNY11 (drought), and the GNY3/GNY12 (water-logging) datasets were each assembled separately using Trinity.

\section{Illumina-oases/velvet}

The 179148376 Illumina reads of length 90 from the 2nd and 3rd replicates of the RNASeq experiments were assembled using Velvet 1.2.03 followed by Oases 0.2.06
[86] with parameters '-min_trans_lgth 200' and '-cov_cutoff auto'. Multiple values of $\mathrm{k}$ were investigated.

\section{Clustering of the transcriptomes}

The three resulting assemblies, namely 454Isotigs, IlluminaTrinity, Illumina-Oases/Velvet, were analyzed separately as was a merge of all 3 data sets (Merge). CDHIT [87], Usearch [88] and TGICL [89] were used to cluster the Merge assembly and the Trinity assembly. Based on performance, TGICL was chosen for the clustering. With TGICL either the default parameters were used or various percent identities were employed as reported. Usearch version 5.2.32 was used with various percentage identities as reported. Cd-hit-est (version 4.5.6-2011-09-02 was used with options ' $-\mathrm{r} 1-\mathrm{M} 10000-\mathrm{T} 6-\mathrm{d} 0-\mathrm{n} 5$ ' as well as several values for the percent identity (option ' $-\mathrm{c}$ '). After preliminary analysis, two transcriptomes were pursuedthe Extended transcriptome (the Merge assembly clustered with TGICL) and the 454-Newbler (the Newbler assembly unclustered).

\section{Coverage tests}

Genomes and Transcriptomes were compared using blastx with E-value 1e-10. The method frac_aligned of the MapTiling Bioperl module was used to estimate the fraction of the tef Sanger sequencing and abiotic stress genes that were aligned to the genome and transcriptomes. The frac_aligned method returns the percentage of the query sequence length that is aligned. As this method filters out any hit with high-scoring segment pairs (HSPs) in more than one context (plus or minus), the coverages generated are underestimates of the total coverage.

\section{Proteome prediction}

Prediction of coding regions of the transcriptomes was accomplished using ESTScan [90] (with $\mathrm{m}=-100, \mathrm{~N}=0$, $\mathrm{w}=60$, and using the Zea mays scoring matrix).

\section{Sequence comparison}

The following databases were obtained from Phytozome [91]: Sorghum bicolor (Phytozome, v. 79) [40], Zea mays (Phytozome, v. 181) [92], Setaria italica (Phytozome, v. 164) [31,93] and Brachypodium distachyon (Phytozome, v. 192) [94]. The Oryza sativa genome was retrieved from IRGSP (version 1.0, 2011-12-05) [95,96].

\section{Phylogenetic trees \\ Waxy tree}

Partial sequences of the WAXY gene from barley (Hordeum vulgare, X07931), bread wheat (Triticum aestivum, KF861808), finger millet (Eleusine coracana, AY508652), foxtail millet (Setaria italica, AB089143), maize (Zea mays, EU041692), Paspalum simplex (AF318770), pearl 
millet (Pennisetum glaucum, AF488414), proso millet (Panicum miliaceum, GU199268), rice (Oryza sativa, FJ235770.1), sorghum (Sorghum bicolor, EF089839), and tef (Eragrostis tef, AY136939) were obtained from the NCBI database. The WAXY sequences were aligned using Mafft (L-INS-I) [97] with the default settings $[98,99]$. PhyML [100] was used to obtain a maximum likelihood tree using the default model of HKY85 + G. Branch support was inferred using the ShimodairaHasegawa-like (SH) aLRT provided by PhyML. Trees were visualized using FigTree v1.3.1 [101].

\section{Supergene tree}

OrthoMCL [102] was used to find homologous gene families between the transcriptome and the entire set of available grass genomes (sorghum, rice, Brachypodium, Setaria and maize). The default parameters (blastp Evalue $<=10^{-5}$ were used. Families with only one ortholog per genome were found and their sequences were aligned for each family. Gapped regions at the beginning and at the end of the alignment were trimmed. The aligned sequences were then concatenated to form 'supergenes' from which the phylogeny and branch lengths were inferred.

\section{SSR tree}

The phylogeny of the SSR sequences was computed with DNAPars from the Phylip package [103] implemented on the T-rex server [104] and visualized with Archaeopteryx [105].

\section{Prolamin tree}

The sequences of the prolamins are based on $\mathrm{Xu}$ and Messing [74] and were downloaded from NCBI. The accession numbers of the sequences used are: ABF99054.1, ACN58179.1, ACM68402.1, 2ACM68402.1, XP_004965361.1, ABP64790.1, NP_001105054.1, AAW32936.1, NP_001105056, NP_001145560, NP_001142525, ABP64793, NP_001105058, CAA39254.1, AAW82166.1, ACJ03469.1, gi_6983787, ABY71029.1, AAA32955.1, AEG74428.1, ACF93465.1, AAV86085.1, CAA37729.1, 21833173, Sb02g025490, AAS73289.1, NP_001105337.1, ADD98895.1, 023603 m, AAS67324.1, AAP80642.1, AAP31050.1, ACR61557.1, AAT93857.1, EES19761.1. The phylogeny of the prolamins was computed using PhyML [100] as implemented on phylogeny.fr [106] and visualized using Archaeopteryx [105].

\section{Sequence analysis}

All multiple sequence alignments were performed using Mafft v6.903b [97] unless otherwise specified. Nucleotidepeptide matches were made with the NucPepMatch package of the Darwin software system [107]. EMBOSS version 6.3.1 was used for sequence manipulation [108].

\section{Estimation of genome size}

The distribution of k-mer frequencies was estimated with jellyfish [37] using $100 \mathrm{bp}$ reads from the $300 \mathrm{bp}$ insert-size library. Jellyfish count was run on fastq files converted to fasta files and options '-s 1000000000-C -c 4'. Jellyfish merge and histo were used to merge the count files and create a histogram, respectively. The maximum of this distribution is related to the sequencing depth $(\mathrm{N})$, read length (L) and kmer length(K) via $\mathrm{M}=\mathrm{N} *(\mathrm{~L}-\mathrm{K}+1) / \mathrm{L}$. The total sequencing depth divided by the real sequencing depth is an estimate of the genome size. The integer value of $M$ from the distribution was used.

\section{SSR detection}

The MISA perl script [109] was used to identify SSRs with the default settings: searching for repeats of mononucleotides that occur more than 10 times, dinucleotides that occur more than six times, and nucleotides of lengths 3-6 which occur more than five times. Perl scripts provided on the MISA website to interface MISA with Primer3 [53,54] were used to design primers for all SSRs with repeat size of three or more (Additional file 6: Table S18 for the entire genome).

\section{Annotation}

Annotation was performed using the Praise internal automated annotation platform of the Swiss Institute of Bioinformatics, a system designed to provide high quality merging of automatic annotations using manually curated rules. It includes annotation extracted from HAMAP [110], PROSITE [111], transmembrane regions, signal peptides as well as the UniProt Feature Table (FT), comments (CC), descriptions (DE) and keywords $(\mathrm{KW})$. It propagates detailed functional annotation (e.g. active site positions) derived from these sources, resolves redundant or conflicting predictions and aggregates all generated annotations into UniProtKB/Swiss-Prot format entries. What could not be annotated by Praise, was then annotated by more traditional method of blasting against UniProt [112], InterPro [113] and PRIAM [114]. Blasting was performed with an E-value of 1e-09.

\section{Abiotic stress gene analysis}

The sequences of 26 genes implicated in abiotic stress in Arabidopsis thaliana, Oryza sativa or Sorghum bicolor were downloaded from NCBI and used to find the protein sequence of the sorghum homolog from the proteome of Sorghum bicolor (Phytozome, version 79) using blastx. Then tblastn was used to search each sorghum abiotic stress protein sequence in the genome and transcriptomes of Eragrostis tef, Zea mays, Sorghum bicolor, Oryza sativa, Brachypodium distrachyum and Setaria italica using a blast E-value of 1e-05. The number of 
copies found with length greater than or equal to $70 \%$ of the length of the query sequence was recorded.

\section{Analysis of gluten-related genes}

Gluten epitopes from wheat (Tritium aestivum), barley (Hordeum vulgare) and rye (Secale cereal) found in TyeDin et al. [5] were sought in the Maker-predicted protein sequences of tef (Eragrostis tef), Brachypodium (Brachypodium distrachyum) version 192, barley (Hordeum vulgare) MIPS vesion 23 March 2012, rice (Oryza sativa) IRGSP version 1.0, 2011-12-05, sorghum (Sorghum bicolor) Phytozome version 79, foxtail millet (Setaria italica) Phytozome version 164 and maize (Zea mays) Phytozome version 181 using MUMmer 3.0 [115]. Exact matches of the 20-amino acid oligopeptide epitopes, core 16-amino acid oligopeptide epitopes, core 13-amino acid oligopeptide epitopes, core 12-amino acid oligopeptide epitopes and core 11-amino acid oligopeptide epitopes were counted. Gluten epitopes were searched in rye (Secale cereal, taxid:4550) and wheat (Tritium aestivum, taxid:4565) using blastP from NCBI BLAST [116] as the whole genomes are unavailable. Nucleotide-peptide matches were obtained with the Darwin [107] bioinformatics platform.

\section{Visualizazion}

The circular plot showing the correspondance betweeen the genetic and physical maps was created with Circos [117]. The comparison of orthologous syntenic genomic regions of the SAL1 gene was created with CoGe $[43,45]$ as was the dotplot of the syntenic map in Figure 2. Trees were visualized using FigTree v1.3.1 [101]. Gepard [46] was used to create the dotplot of the A and B genomes.

\section{Availability of supporting data}

Data, analyses and updates can be downloaded from: http://www.tef-research.org/genome.html.

The genome and maker annotations are available for viewing, blasting and download at CoGe at: https:// genomevolution.org/CoGe/GenomeInfo.pl?gid=22790.

The project is registered at NCBI with BioProject Number: http://www.ncbi.nlm.nih.gov/bioproject/253673.

The sample accessions are: SAMN02872936 (for GNY7); SAMN02872919 (GNY8); GNY9 and SAMN02872920 (GNY9).

Accession numbers in the Sequence Read Archive are: SRR1463375, SRR1463376, SRR1463377, SRR1463396, SRR1463397 and SRR1463402.

\section{Additional files}

Additional file 1: Figure S1. Alignment of tef $\mathrm{KO} 2 \mathrm{~A}$ and $\mathrm{B}$ copies. Additional file 2: A file with Supplementary Notes, Supplementary Figures and Supplementary Tables and references.
Additional file 3: Table S7. Location of SSR markers in the tef genome.

Additional file 4: Figure S4. Alignment of A and B genomes.

Additional file 5: Table S12. Location of tef CNLT markers in the pseudo-chromosomes of tef ordered by linkage group.

Additional file 6: Table S18. List of selected SSRs identified from scaffolds.

\section{Abbreviations}

aLRT: Approximate likelihood ratio test; bp: Base pair; BLAST: Basic local alignment search tool; CC: Comments; CDS: Coding sequence;

CoGe: Comparative genomics platform; DE: Descriptions; ERD1: Early responsive to dehydration stress 1; EST: Expressed sequence tags; FT: Feature table; Gbp: Giga base pair; GBS: Genotyping by sequencing; Gl: Glycemic index; GWAS: Genome wide association studies, HSPs, high-scoring segment pairs; IRGSP: International rice genome sequencing project; Ka: Non-synonymous substitution rate; kbp: Kilo base pair; Ks: Synonymous substitution rate; KW: Key word; LMW: Low-molecular-weight subunit; Mbp: Mega base pair; MIPS: Munich information center for protein sequences; MISA: MIcro SAtellite identification tool; MITEs: Miniature inverted-repeat transposable elements; ML: Maximum likelihood; MYA: Million years ago; NCBI: National Center for Biotechnology Information; PAML: Phylogenetic analysis by maximum likelihood; PRIAM: PRofils pour I'Identification Automatique du Métabolisme (enzyme-specific profiles for metabolic pathway prediction); SGR: Stay green rice; SSR: Simple sequence repeats; TE: Transposable elements; TGICL: TIGR Gene indices clustering tools; TREP: Triticeae repeat sequence database.

\section{Competing interests}

The authors declare they have no competing interests.

\section{Authors' contributions}

GC, SC, KA; CK and ZT conceived and designed experiments; LFar: performed genome sequencing; SPW, SL, RB and ZT performed lab experiments; GC, SPW, KE, YSW, DG, EdC, EL, MS and LFal performed analyses; GC and ZT wrote the manuscript; SPW, KE, DG, SC, EL, KA and CK edited the manuscript; All authors read and approved the final manuscript.

\section{Acknowledgements}

We thank Nigussu Husein from the Debre Zeit Research Center in Ethiopia, Eligio Bossolini, Abiel Rindisbacher, Christopher Ball, Nicole Signer and Jasmin Sekulovski from the Institute of Plant Sciences, University of Bern for their technical help. We also thank Syngenta Foundation for Sustainable Agriculture, SystemsX.ch and University of Bern for financial support. The computations were performed at the Vital-IT Center for high-performance computing of the SIB Swiss Institute of Bioinformatics.

\section{Author details}

${ }^{1}$ University of Bern, Institute of Plant Sciences, Altenbergrain 21, Bern $\mathrm{CH}-3013$, Switzerland. ${ }^{2}$ Swiss Institute of Bioinformatics, Vital-IT, Quartier Sorge - Batiment Genopode, Lausanne 1015, Switzerland. ${ }^{3}$ Ethiopian Institute of Agricultural Research, National Biotechnology Laboratory (Holetta), P.O. Box 2003, Addis Ababa, Ethiopia. ${ }^{4}$ Swiss Institute of Bioinformatics, Rue Michel-Servet 1, 1211, Geneva 4, Switzerland. ${ }^{5}$ Ethiopian Institute of Agricultural Research, Debre Zeit Agricultural Research Center, P.O. Box 32 , Debre Zeit, Ethiopia. ${ }^{6}$ Fasteris SA, Ch. du Pont-du-Centenaire 109, P.O. Box 28, Plan-les-Ouates CH-1228, Switzerland. 'Univerisity of Arizona, School of Plant Sciences, 1140 E. South Campus Drive, 303 Forbes Building, P.O. Box 210036, Tucson, AZ 85721-0036, USA. ${ }^{8}$ Current address: University of Bern, Clinic for Parodontology, Freiburgstrasse 7, Bern CH-3010, Switzerland. ${ }^{9}$ Current address: University of Fribourg, Faculty of Science, Ch. du Musée 10, Fribourg $\mathrm{CH}-1700$, Switzerland.

Received: 27 March 2014 Accepted: 3 July 2014

Published: 9 July 2014

\section{References}

1. CSA: Agricultural Sample Survey for 2012/13. Ethiopia: Statistical Bulletin Addis Ababa; 2013. 
2. Umeta $M$, West $C E$, Fufa $H$ : Content of zinc, iron, calcium and their absorption inhibitors in foods commonly consumed in Ethiopia. J Food Compos Anal 2005, 18(8):803-817.

3. Eragrain. [http://www.eragrain.com/pdf/Consumer\%20brochure\%2052012\%20no\%20address\%20label.pdf]

4. Alaunyte I, Stojceska V, Plunkett A, Ainsworth P, Derbyshire E: Improving the quality of nutrient-rich Teff (Eragrostis tef) breads by combination of enzymes in straight dough and sourdough breadmaking. J Cereal Sci 2012, 55(1):22-30.

5. Tye-Din JA, Stewart JA, Dromey JA, Beissbarth T, van Heel DA, Tatham A Henderson K, Mannering SI, Gianfrani C, Jewell DP, Hill AV, McCluskey J, Rossjohn J, Anderson RP: Comprehensive, quantitative mapping of T cell epitopes in gluten in celiac disease. Sci Trans/ Med 2010, 2(41):41 ra51.

6. Hopman E, Dekking L, Blokland ML, Wuisman M, Zuijderduin W, Koning F, Schweizer J: Tef in the diet of celiac patients in The Netherlands. Scand J Gastroenterol 2008, 43(3):277-282

7. Spaenij-Dekking L, Kooy-Winkelaar Y, Koning F: The Ethiopian cereal tef in celiac disease. New Engl J Med 2005, 353(16):1748-1749.

8. Zeid M, Belay G, Mulkey S, Poland J, Sorrells ME: QTL mapping for yield and lodging resistance in an enhanced SSR-based map for tef. Theor App/ Genet 2011, 122(1):77-93.

9. Yu JK, Sun Q, Rota ML, Edwards H, Tefera H, Sorrells ME: Expressed sequence tag analysis in tef (Eragrostis tef (Zucc) Trotter). Genome 2006, 49(4):365-372

10. Smith SM, Yuan YN, Doust AN, Bennetzen JL: Haplotype analysis and linkage disequilibrium at five loci in eragrostis tef. G3-Genes Genom Genet 2012, 2(3):407-419.

11. Ingram AL, Doyle JJ: The origin and evolution of Eragrostis tef (Poaceae) and related polyploids: Evidence from nuclear waxy and plastid rps16. Am J Bot 2003, 90(1):116-122

12. Bekele $\mathrm{E}$, Lester RN: Biochemical assessment of the relationships of eragrostis-Tef (Zucc) Trotter with Some Wild Eragrostis Species (Gramineae). Ann Bot-London 1981, 48(5):717-725.

13. Hundera F, Arumuganathan K, Baenziger P: Determination of relative nuclear DNA content of tef [Eragrostis tef (Zucc.) Trotter] using flow cytometry. J Genet Breed 2000, 54:165-168.

14. Soltis PS, Soltis DE: The role of hybridization in plant speciation. Annu Rev Plant Biol 2009, 60:561-588

15. Van de Peer $Y$, Maere S, Meyer A: OPINION The evolutionary significance of ancient genome duplications. Nat Rev Genet 2009, 10(10):725-732.

16. Brochmann C, Brysting AK, Alsos IG, Borgen L, Grundt HH, Scheen AC, Elven R: Polyploidy in arctic plants. Biol J Linn Soc 2004, 82(4):521-536.

17. Leitch AR, Leitch IJ: Perspective - genomic plasticity and the diversity of polyploid plants. Science 2008, 320(5875):481-483

18. Schatz MC, Witkowski J, McCombie WR: Current challenges in de novo plant genome sequencing and assembly. Genome Biol 2012, 13(4):243.

19. Phillippy AM, Schatz MC, Pop M: Genome assembly forensics: finding the elusive mis-assembly. Genome Biol 2008, 9(3):R55.

20. Kelley DR, Salzberg SL: Detection and correction of false segmental duplications caused by genome mis-assembly. Genome Biol 2010, 11(3):R28

21. Yukawa M, Tsudzuki T, Sugiura M: The chloroplast genome of Nicotiana sylvestris and Nicotiana tomentosiformis: complete sequencing confirms that the Nicotiana sylvestris progenitor is the maternal genome donor of Nicotiana tabacum. Mol Genet Genomics 2006, 275(4):367-373.

22. Wang K, Wang Z, Li F, Ye W, Wang J, Song G, Yue Z, Cong L, Shang H, Zhu S, Zou C, Li Q, Yuan Y, Lu C, Wei H, Gou C, Zheng Z, Yin Y, Zhang X, Liu K, Wang B, Song C, Shi N, Kohel RJ, Percy RG, Yu JZ, Zhu YX, Wang J, Yu S: The draft genome of a diploid cotton Gossypium raimondii. Nat Genet 2012, 44(10):1098-1103.

23. Wurm Y, Wang J, Riba-Grognuz O, Corona M, Nygaard S, Hunt BG, Ingram KK, Falquet L, Nipitwattanaphon M, Gotzek D, Dijkstra MB, Oettler J, Comtesse F, Shih CJ, Wu WJ, Yang CC, Thomas J, Beaudoing E, Pradervand S, Flegel V, Cook ED, Fabbretti R, Stockinger H, Long L, Farmerie WG, Oakey J, Boomsma JJ, Pamilo P, Yi SV, Heinze J, et al: The genome of the fire ant Solenopsis invicta. Proc Natl Acad Sci U S A 2011, 108(14):5679-5684.

24. Consortium PGS: Genome sequence and analysis of the tuber crop potato. Nature 2011, 475(7355):U189-U194.

25. Schreiber AW, Hayden MJ, Forrest KL, Kong SL, Langridge P, Baumann U: Transcriptome-scale homoeolog-specific transcript assemblies of bread wheat. BMC Genomics 2012, 13:492
26. Luo R, Liu B, Xie Y, Li Z, Huang W, Yuan J, He G, Chen Y, Pan Q, Liu Y, Tang J, Wu G, Zhang H, Shi Y, Liu Y, Yu C, Wang B, Lu Y, Han C, Cheung DW, Yiu SM, Peng S, Xiaoqian Z, Liu G, Liao X, Li Y, Yang H, Wang J, Lam TW, Wang J: SOAPdenovo2: an empirically improved memory-efficient short-read de novo assembler. GigaScience 2011, 1(1):18-18.

27. Margulies M, Egholm M, Altman WE, Attiya S, Bader JS, Bemben LA, Berka J, Braverman MS, Chen YJ, Chen Z, Dewell SB, Du L, Fierro JM, Gomes XV, Godwin BC, He W, Helgesen S, Ho CH, Irzyk GP, Jando SC, Alenquer ML, Jarvie TP, Jirage KB, Kim JB, Knight JR, Lanza JR, Leamon JH, Lefkowitz SM, Lei $M$, Li J, et al: Genome sequencing in microfabricated high-density picolitre reactors. Nature 2005, 437(7057):376-380.

28. Grabherr MG, Haas BJ, Yassour M, Levin JZ, Thompson DA, Amit I, Adiconis X, Fan L, Raychowdhury R, Zeng Q, Chen Z, Mauceli E, Hacohen N, Gnirke A, Rhind N, di Palma F, Birren BW, Nusbaum C, Lindblad-Toh K, Friedman N, Regev A: Full-length transcriptome assembly from RNA-Seq data without a reference genome. Nat Biotechnol 2011, 29(7):644-652.

29. Zerbino DR, Birney E: Velvet: Algorithms for de novo short read assembly using de Bruijn graphs. Genome Res 2008, 18(5):821-829.

30. Nakasugi $K$, Crowhurst R, Bally J, Waterhouse P: Combining transcriptome assemblies from multiple de novo assemblers in the allo-tetraploid plant nicotiana benthamiana. PLOS ONE 2014, 9(3):e91776. doi:10.1371/journal. pone.0091776.

31. Bennetzen JL, Schmutz J, Wang H, Percifield R, Hawkins J, Pontaroli AC Estep M, Feng L, Vaughn JN, Grimwood J, Jenkins J, Barry K, Lindquist E, Hellsten U, Deshpande S, Wang X, Wu X, Mitros T, Triplett J, Yang X, Ye CY, Mauro-Herrera M, Wang L, Li P, Sharma M, Sharma R, Ronald PC, Panaud O, Kellogg EA, Brutnell TP, et al: Reference genome sequence of the model plant Setaria. Nat Biotechnol 2012, 30(6):555-561.

32. Zhang G, Liu X, Quan Z, Cheng S, Xu X, Xie M, Zeng P, Yue Z, Wang W, Tao Y, Bian C, Han C, Xia Q, Peng X, Cao R, Yang X, Zhan D, Hu J, Zhang Y, Li H, Li H, Li N, Wang J, Wang C, Wang R, Guo T, Cai Y, Liu C, Xiang H, Shi Q, et al: Genome sequence of foxtail millet (Setaria italica) provides insights into grass evolution and biofuel potential. Nat Biotechnol 2012, 30(6):549-554.

33. Huang S, Li R, Zhang Z, Li L, Gu X, Fan W, Lucas WJ, Wang X, Xie B, Ni P. Ren Y, Zhu H, Li J, Lin K, Jin W, Fei Z, Li G, Staub J, Kilian A, van der Vossen EA, Wu Y, Guo J, He J, Jia Z, Ren Y, Tian G, Lu Y, Ruan J, Qian W, Wang M, et al: The genome of the cucumber, cucumis sativus L. Nat Genet 2009, 41(12):1275-1281.

34. Guo S, Zhang J, Sun $H$, Salse J, Lucas WJ, Zhang H, Zheng Y, Mao L, Ren Y, Wang Z, Min J, Guo X, Murat F, Ham BK, Zhang Z, Gao S, Huang M, Xu Y, Zhong S, Bombarely A, Mueller LA, Zhao H, He H, Zhang Y, Zhang Z, Huang $S$, Tan T, Pang E, Lin K, Hu Q, et al: The draft genome of watermelon (Citrullus lanatus) and resequencing of 20 diverse accessions. Nat Genet 2012, 45(1):51-58.

35. Argout X, Salse J, Aury JM, Guiltinan MJ, Droc G, Gouzy J, Allegre M, Chaparro C, Legavre T, Maximova SN, Abrouk M, Murat F, Fouet O, Poulain J, Ruiz M, Roguet Y, Rodier-Goud M, Barbosa-Neto JF, Sabot F, Kudrna D, Ammiraju JSS, Schuster SC, Carlson JE, Sallet E, Schiex T, Dievart A, Kramer M, Gelley L, Shi Z, Berard A, et al: The genome of Theobroma cacao. Nat Genet 2010, 43(2):101-108

36. Al-Mssallem IS, Hu S, Zhang X, Lin Q, Liu W, Tan J, Yu X, Liu J, Pan L, Zhang T, Yin Y, Xin C, Wu H, Zhang G, Ba Abdullah MM, Huang D, Fang Y, Alnakhli YO, Jia S, Yin A, Alhuzimi EM, Alsaihati BA, Al-Owayyed SA, Zhao D, Zhang S, Al-Otaibi NA, Sun G, Majrashi MA, Li F, Tala: De novo genome sequencing and comparative genomics of date palm (Phoenix dactylifera). Nat Biotechnol 2011, 29(6):521-527.

37. Marcais G, Kingsford C: A fast, lock-free approach for efficient parallel counting of occurrences of k-mers. Bioinformatics 2011, 27(6):764-770.

38. Li XM, Waterman MS: Estimating the repeat structure and length of DNA sequences using I-tuples. Genome Res 2003, 13(8):1916-1922.

39. Li R, Fan W, Tian G, Zhu H, He L, Cai J, Huang Q, Cai Q, Li B, Bai Y, Zhang Z, Zhang Y, Wang W, Li J, Wei F, Li H, Jian M, Li J, Zhang Z, Nielsen R, Li D, Gu W, Yang Z, Xuan Z, Ryder OA, Leung FC, Zhou Y, Cao J, Sun X, Fu Y, et al: The sequence and de novo assembly of the giant panda genome (vol 463, pg 311, 2010). Nature 2010, 463(7284):1106-1106.

40. Paterson AH, Bowers JE, Bruggmann R, Dubchak I, Grimwood J, Gundlach H, Haberer G, Hellsten U, Mitros T, Poliakov A, Schmutz J, Spannagl M, Tang H, Wang X, Wicker T, Bharti AK, Chapman J, Feltus FA, Gowik U, Grigoriev IV, Lyons E, Maher CA, Martis M, Narechania A, Otillar RP, Penning BW, Salamov AA, Wang Y, Zhang L, Carpita NC, et al: The Sorghum bicolor genome and the diversification of grasses. Nature 2009, 457(7229):551-556. 
41. Holt C, Yandell M: MAKER2: an annotation pipeline and genomedatabase management tool for second-generation genome projects. BMC Bioinformatics 2011, 12(1):491. doi:10.1186/1471-2105-12-491.

42. Cantarel BL, Korf I, Robb SM, Parra G, Ross E, Moore B, Holt C, Sanchez Alvarado A, Yandell M: MAKER: an easy-to-use annotation pipeline designed for emerging model organism genomes. Genome Res 2008, 18(1):188-196

43. Lyons E, Freeling M: How to usefully compare homologous plant genes and chromosomes as DNA sequences. Plant J 2008, 53(4):661-673.

44. Lyons E, Pedersen B, Kane J, Freeling M: The value of nonmodel genomes and an example using SynMap Within CoGe to dissect the hexaploidy that predates the rosids. Trop Plant Biol 2008, 1(3-4):181-190.

45. Lyons E, Pedersen B, Kane J, Alam M, Ming R, Tang H, Wang X, Bowers J, Paterson A, Lisch D, Freeling M: Finding and comparing syntenic regions among arabidopsis and the outgroups papaya, poplar, and grape: CoGe with rosids. Plant Physiol 2008, 148(4):1772-1781.

46. Krumsiek J, Arnold R, Rattei T: Gepard: a rapid and sensitive tool for creating dotplots on genome scale. Bioinformatics 2007, 23(8):1026-1028.

47. Zeid M, Belay G, Mulkey S, Poland J, Sorrells ME: QTL mapping for yield and lodging resistance in an enhanced SSR-based map for tef. Theor Appl Genet 2010, 122(1):77-93.

48. Yang Z: PAML 4: phylogenetic analysis by maximum likelihood. $\mathrm{Mol}$ Biol Evol 2007, 24(8):1586-1591.

49. Gaut BS, Morton BR, McCaig BC, Clegg MT: Substitution rate comparisons between grasses and palms: Synonymous rate differences at the nuclear gene Adh parallel rate differences at the plastid gene rbcL. Proc Natl Acad Sci U S A 1996, 93(19):10274-10279.

50. Swigonova Z, Lai JS, Ma JX, Ramakrishna W, Llaca V, Bennetzen JL, Messing $\mathrm{J}$ : Close split of sorghum and maize genome progenitors. Genome Res 2004, 14(10A):1916-1923.

51. Paterson AH, Bowers JE, Chapman BA: Ancient polyploidization predating divergence of the cereals, and its consequences for comparative genomics. Proc Natl Acad Sci U S A 2004, 101(26):9903-9908.

52. MISA - MlcroSAtellite identification tool. [http://pgrc.ipk-gatersleben.de/misa/]

53. Untergasser A, Cutcutache I, Koressaar T, Ye J, Faircloth BC, Remm M, Rozen SG: Primer3-new capabilities and interfaces. Nucleic Acids Res 2012, 40(15):e115. doi:10.1093/nar/gks596.

54. Koressaar T, Remm M: Enhancements and modifications of primer design program Primer3. Bioinformatics 2007, 23(10):1289-1291.

55. Peng J, Richards DE, Hartley NM, Murphy GP, Devos KM, Flintham JE, Beales J, Fish LJ, Worland AJ, Pelica F, Sudhakar D, Christou P, Snape JW, Gale MD, Harberd NP: 'Green revolution' genes encode mutant gibberellin response modulators. Nature 1999, 400(6741):256-261.

56. Plaza-Wüthrich S, Cannarozzi G, Tadele Z: Genetic and phenotypic diversity in selected genotypes of tef [Eragrostis tef (Zucc.)] Trotter. Afr J Agr Res 2013, 8(12):1041-1049.

57. Assefa K, Aliye S, Belay G, Metaferia G, Tefera H, Sorrells ME: Quncho: the first popular tef variety in Ethiopia. Int J Agr Sustain 2011, 9(1):25-34.

58. Wicker T, Matthews DE, Keller B: TREP: a database for Triticeae repetitive elements. Trends Plant Sci 2002, 7(12):561-562.

59. Salzberg SL, Yorke JA: Beware of mis-assembled genomes. Comput Appl Biosci 2005, 21(24):4320-4321.

60. Nawrocki EP, Kolbe DL, Eddy SR: Infernal 1.0: inference of RNA alignments. Bioinformatics 2009, 25(10):1335-1337.

61. Gardner PP, Daub J, Tate JG, Nawrocki EP, Kolbe DL, Lindgreen S, Wilkinson AC, Finn RD, Griffiths-Jones S, Eddy SR, Bateman A: Rfam: updates to the RNA families database. Nucleic Acids Res 2009, 37(Database):D136-D140.

62. Griffiths-Jones S, Moxon S, Marshall M, Khanna A, Eddy SR, Bateman A: Rfam: annotating non-coding RNAs in complete genomes. Nucleic Acids Res 2005, 33(Database issue):D121-D124.

63. Chan PP, Lowe TM: GtRNAdb: a database of transfer RNA genes detected in genomic sequence. Nucleic Acids Res 2009, 37:D93-D97.

64. Han Y, Wessler SR: MITE-Hunter: a program for discovering miniature inverted-repeat transposable elements from genomic sequences. Nucleic Acids Res 2010, 38(22):e199-e199.

65. Oki N, Yano K, Okumoto Y, Tsukiyama T, Teraishi M, Tanisaka T: A genomewide view of miniature inverted-repeat transposable elements (MITEs) in rice. Oryza sativa ssp japonica Genes Genet Syst 2008, 83(4):321-329.

66. Assefa K, Yu JK, Zeid M, Belay G, Tefera H, Sorrells ME: Breeding tef [Eragrostis tef (Zucc.) trotter]: conventional and molecular approaches. Plant Breed 2011, 130(1):1-9.
67. ABCIC: Effects Of Climate Change On Eragrostis Tef In Ethiopia: A Call For Action To Avert Food Security Crisis. In ABCIC Policy Brief No1. 2011.

68. Wilson PB, Estavillo GM, Field KJ, Pornsiriwong W, Carroll AJ, Howell KA, Woo NS, Lake JA, Smith SM, Harvey Millar A, von Caemmerer S, Pogson BJ: The nucleotidase/phosphatase SAL1 is a negative regulator of drought tolerance in Arabidopsis. Plant J 2009, 58(2):299-317.

69. Manmathan H, Shaner D, Snelling J, Tisserat N, Lapitan N: Virus-induced gene silencing of Arabidopsis thaliana gene homologues in wheat identifies genes conferring improved drought tolerance. J Exp Bot 2013, 64(5):1381-1392

70. Akiyama T, Pillai MA: Molecular cloning, characterization and in vitro expression of a novel endo-1,3-beta-glucanase up-regulated by ABA and drought stress in rice (Oryza sativa L.). Plant Sci 2001, 161(6):1089-1098.

71. Jiang H, Li M, Liang N, Yan H, Wei Y, Xu X, Liu J, Xu Z, Chen F, Wu G: Molecular cloning and function analysis of the stay green gene in rice. Plant J 2007, 52(2):197-209.

72. Nakashima K, Kiyosue T, YamaguchiShinozaki K, Shinozaki K: A nuclear gene, erd1 encoding a chloroplast-targeted Clp protease regulatory subunit homolog is not only induced by water stress but also developmentally up-regulated during senescence in Arabidopsis thaliana. Plant J 1997, 12(4):851-861

73. Altschul SF, Gish W, Miller W, Myers EW, Lipman DJ: Basic Local Alignment Search Tool. J Mol Biol 1990, 215(3):403-410.

74. $\mathrm{Xu} \mathrm{JH}$, Messing J: Amplification of prolamin storage protein genes in different subfamilies of the Poaceae. Theor Appl Genet 2009,

119(8):1397-1412.

75. Tatham AS, Fido RJ, Moore CM, Kasarda DD, Kuzmicky DD, Keen JN, Shewry PR: Characterisation of the major prolamins of tef (Eragrostis tef) and finger millet (Eleusine coracana). J Cereal Sci 1996, 24(1):65-71.

76. Fasteris - DNA Sequencing Service - Swiss quality. [https://www.fasteris.com/]

77. Functional Genomics Center Zurich. [http://www.fgcz.ch/]

78. Advancing through genomics. [http://www.macrogen.com/eng/]

79. FastQC. [http://www.bioinformatics.babraham.ac.uk/projects/fastqc/]

80. Li R, Zhu H, Ruan J, Qian W, Fang X, Shi Z, Li Y, Li S, Shan G, Kristiansen K, Li S, Yang $\mathrm{H}$, Wang J, Wang J: De novo assembly of human genomes with massively parallel short read sequencing. Genome Res 2010, 20(2):265-272.

81. Morgulis A, Gertz EM, Schäffer AA, Agarwala R: WindowMasker: windowbased masker for sequenced genomes. Bioinformatics 2006, 22(2):134-141.

82. RepeatMasker Open-3.0. [http://www.repeatmasker.org]

83. Stanke M, Keller O, Gunduz I, Hayes A, Waack S, Morgenstern B: AUGUSTUS: ab initio prediction of alternative transcripts. Nucleic Acids Res 2006, 34:W435-W439.

84. Korf I: Gene finding in novel genomes. BMC Bioinformatics 2004, 5:59 doi:10.1186/1471-2105-5-59.

85. Lowe TM, Eddy SR: tRNAscan-SE: a program for improved detection of transfer RNA genes in genomic sequence. Nucleic Acids Res 1997, 25(5):0955-0964.

86. Schulz MH, Zerbino DR, Vingron M, Birney E: Oases: robust de novo RNA-seq assembly across the dynamic range of expression levels. Bioinformatics 2012, 28(8):1086-1092.

87. Fu L, Niu B, Zhu Z, Wu S, Li W: CD-HIT: accelerated for clustering the nextgeneration sequencing data. Comput Appl Biosci 2012, 28(23):3150-3152.

88. Edgar RC: Search and clustering orders of magnitude faster than BLAST. Comput Appl Biosci 2010, 26(19):2460-2461.

89. Pertea G, Huang X, Liang F, Antonescu V, Sultana R, Karamycheva S, Lee $Y$, White J, Cheung F, Parvizi B, Tsai J, Quackenbush J: TIGR Gene Indices clustering tools (TGICL): a software system for fast clustering of large EST datasets. Bioinformatics 2003, 19(5):651-652.

90. Iseli C, Jongeneel CV, Bucher P: ESTScan: a program for detecting, evaluating, and reconstructing potential coding regions in EST sequences. Proc Int Conf Intell Syst Mol Biol 1999, 7:138-148.

91. Goodstein DM, Shu S, Howson R, Neupane R, Hayes RD, Fazo J, Mitros T, Dirks W, Hellsten U, Putnam N, Rokhsar DS: Phytozome: a comparative platform for green plant genomics. Nucleic Acids Res 2012, 40(D1):D1178-D1186.

92. Schnable PS, Ware D, Fulton RS, Stein JC, Wei FS, Pasternak S, Liang CZ, Zhang JW, Fulton L, Graves TA, Minx P, Reily AD, Courtney L, Kruchowski SS, Tomlinson C, Strong C, Delehaunty K, Fronick C, Courtney B, Rock SM, Belter E, Du F, Kim K, Abbott RM, Cotton M, Levy A, Marchetto P, Ochoa K, Jackson SM, Gillam B, et al: The B73 maize genome: complexity, diversity, and dynamics. Science 2009, 326(5956):1112-1115. 
93. Doust AN, Kellogg EA, Devos KM, Bennetzen JL: Foxtail millet: a sequencedriven grass model system. Plant Physiol 2009, 149(1):137-41.

94. Vogel JP, Garvin DF, Mockler TC, Schmutz J, Rokhsar D, Bevan MW, Barry K, Lucas S, Harmon-Smith M, Lail K, Tice H, Schmutz J, Grimwood J, McKenzie N, Bevan MW, Huo N, Gu YQ, Lazo GR, Anderson OD, Vogel JP, You FM, Luo MC, Dvorak J, Wright J, Febrer M, Bevan MW, Idziak D, Hasterok R, Garvin $D F$, Lindquist $E$, et al: Genome sequencing and analysis of the model grass Brachypodium distachyon. Nature 2010, 463(7282):763-768.

95. Sakai H, Lee SS, Tanaka T, Numa H, Kim J, Kawahara Y, Wakimoto H, Yang C, Iwamoto M, Abe T, Yamada Y, Muto A, Inokuchi H, Ikemura T, Matsumoto T, Sasaki T, Itoh T: Rice Annotation Project Database (RAP-DB): an integrative and interactive database for rice genomics. Plant Cell Physiol 2013, 54(2):E6-+.

96. Kawahara Y, de la Bastide M, Hamilton JP, Kanamori H, McCombie WR, Ouyang S, Schwartz DC, Tanaka T, Wu J, Zhou S, Childs KL, Davidson RM, Lin H, Quesada-Ocampo L, Vaillancourt B, Sakai H, Lee SS, Kim J, Numa H, Itoh T, Buell CR, Matsumoto T: Improvement of the Oryza sativa Nipponbare reference genome using next generation sequence and optical map data. Rice 2013, 6:4. doi:10.1186/1939-8433-6-4

97. Katoh K, Kuma K, Toh H, Miyata T: MAFFT version 5: improvement in accuracy of multiple sequence alignment. Nucleic Acids Res 2005, 33(2):511-518

98. Larkin MA, Blackshields G, Brown NP, Chenna R, McGettigan PA, McWilliam $H$, Valentin F, Wallace IM, Wilm A, Lopez R, Thompson JD, Gibson TJ, Higgins DG: Clustal W and Clustal X version 2.0. Bioinformatics 2007, 23(21):2947-2948.

99. Thompson JD, Higgins DG, Gibson TJ: CLUSTAL W: improving the sensitivity of progressive multiple sequence alignment through sequence weighting, position-specific gap penalties and weight matrix choice. Nucleic Acids Res 1994, 22(22):4673-4680

100. Guindon S, Lethiec F, Duroux P, Gascuel O: PHYML Online - a web server for fast maximum likelihood-based phylogenetic inference. Nucleic Acids Res 2005, 33:W557-W559.

101. FigTree. [http://tree.bio.ed.ac.uk/software/figtree/]

102. Li L, Stoeckert CJ, Roos DS: OrthoMCL: identification of ortholog groups for eukaryotic genomes. Genome Res 2003, 13(9):2178-2189.

103. Felsenstein J: PHYLIP - phylogeny inference package (Version 3.2). Cladistics 1989, 5:3

104. Alix B, Boubacar DA, Vladimir M: T-REX: a web server for inferring, validating and visualizing phylogenetic trees and networks. Nucleic Acids Res 2012, 40(W1):W573-W579.

105. Han MV, Zmasek CM: phyloXML: XML for evolutionary biology and comparative genomics. Bmc Bioinformatics 2009, 10:356. doi:10.1186/1471-2105-10-356.

106. Dereeper A, Guignon V, Blanc G, Audic S, Buffet S, Chevenet F, Dufayard JF, Guindon S, Lefort V, Lescot M, Claverie JM, Gascuel O: Phylogeny.fr: robust phylogenetic analysis for the non-specialist. Nucleic Acids Res 2008, 36:W465-W469.

107. Gonnet GH, Hallett MT, Korostensky C, Bernardin L: Darwin v. 2.0: an interpreted computer language for the biosciences. Bioinformatics 2000, 16(2):101-103.

108. Rice P, Longden I, Bleasby A: EMBOSS: the European molecular biology open software suite. Trends Genet 2000, 16(6):276-277.

109. MISA: MlcroSAtellite identification tool. [http://pgrc.ipk-gatersleben.de/misa]

110. Pedruzzi I, Rivoire C, Auchincloss AH, Coudert E, Keller G, de Castro E, Baratin D, Cuche BA, Bougueleret L, Poux S, Redaschi N, Xenarios I, Bridge A: HAMAP in 2013, new developments in the protein family classification and annotation system. Nucleic Acids Res 2013, 2013:584-589.

111. Sigrist CJA, de Castro E, Cerutti L, Cuche BA, Hulo N, Bridge A, Bougueleret $L$, Xenarios I: New and continuing developments at PROSITE. Nucleic Acids Res 2013, 41(D1):E344-E347.

112. Bairoch A, Bougueleret L, Altairac S, Amendolia V, Auchincloss A, Argoud-Puy G, Axelsen K, Baratin D, Blatter MC, Boeckmann B, Bolleman J, Bollondi L, Boutet E, Quintaje SB, Breuza L, Bridge A, de Castro E, Ciapina L, Coral D, Coudert E, Cusin I, Delbard G, Dornevil D, Roggli PD, Duvaud S, Estreicher A, Famiglietti L, Feuermann M, Gehant S, Farriol-Mathis N, et al: The Universal Protein Resource (UniProt) 2009. Nucleic Acids Res 2009, 37:D169-D174.

113. Hunter $S$, Jones $P$, Mitchell A, Apweiler R, Attwood TK, Bateman A, Bernard T, Binns D, Bork P, Burge S, de Castro E, Coggill P, Corbett M, Das U, Daugherty L, Duquenne L, Finn RD, Fraser M, Gough J, Haft D, Hulo N, Kahn D, Kelly E, Letunic I, Lonsdale D, Lopez R, Madera M, Maslen J, McAnulla C, McDowall J, et al: InterPro in 2011: new developments in the family and domain prediction database. Nucleic Acids Res 2012, 40(D1):D306-D312

114. Claudel-Renard C, Chevalet C, Faraut T, Kahn D: Enzyme-specific profiles for genome annotation: PRIAM. Nucleic Acids Res 2003, 31(22):6633-6639.

115. Delcher AL, Salzberg SL, Phillippy AM: Using MUMmer to identify similar regions in large sequence sets. Curr Protoc Bioinformatics 2003, Chapter 10:Unit-Uni3.

116. NCBI/Blast. [http://blast.ncbi.nlm.nih.gov/Blast.cgi?CMD=Web\&PAGE_TYPE= BlastHome]

117. Krzywinski M, Schein J, Birol I, Connors J, Gascoyne R, Horsman D, Jones SJ, Marra MA: Circos: an information aesthetic for comparative genomics. Genome Res 2009, 19(9):1639-1645.

doi:10.1186/1471-2164-15-581

Cite this article as: Cannarozzi et al:: Genome and transcriptome sequencing identifies breeding targets in the orphan crop tef (Eragrostis tef). BMC Genomics 2014 15:581.

\section{Submit your next manuscript to BioMed Central and take full advantage of:}

- Convenient online submission

- Thorough peer review

- No space constraints or color figure charges

- Immediate publication on acceptance

- Inclusion in PubMed, CAS, Scopus and Google Scholar

- Research which is freely available for redistribution

Submit your manuscript at www.biomedcentral.com/submit
C) Biomed Central 